\title{
Validity of abundances derived from spaxel spectra of the MaNGA survey
}

\author{
L. S. Pilyugin ${ }^{1,2,3}$, E. K. Grebel ${ }^{3}$, I. A. Zinchenko ${ }^{2,3}$, Y. A. Nefedyev ${ }^{4}$, V. M. Shulga ${ }^{1,5,6}$, \\ H. Wei ${ }^{1,6}$, and P.P. Berczik ${ }^{1,2,3}$
}

\author{
${ }^{1}$ The International Center of Future Science of the Jilin University, 2699 Qianjin St., 130012 Changchun, PR China \\ ${ }^{2}$ Main Astronomical Observatory, National Academy of Sciences of Ukraine, 27 Akademika Zabolotnoho St, 03680 Kiev, Ukraine \\ e-mail: pilyugin@MAO.Kiev.UA \\ 3 Astronomisches Rechen-Institut, Zentrum für Astronomie der Universität Heidelberg, Mönchhofstr. 12-14, 69120 Heidelberg, \\ Germany \\ ${ }^{4}$ Kazan Federal University, 18 Kremlyovskaya St., 420008 Kazan, Russian Federation \\ ${ }^{5}$ Institut of Radio Astronomy of National Academy of Sciences of Ukraine, 4 Mystetstv str., 61002 Kharkov, Ukraine \\ ${ }^{6}$ College of Physics, The Jilin University, 2699 Qianjin St., 130012 Changchun, PR China
}

Received 27 October 2017 / Accepted 27 November 2017

\begin{abstract}
We measured the emission lines in the spaxel spectra of Mapping Nearby Galaxies at Apache Point Observatory (MaNGA) galaxies in order to determine the abundance distributions therein. It has been suggested that the strength of the low-ionization lines, $\mathrm{R}_{2}, \mathrm{~N}_{2}$, and $\mathrm{S}_{2}$, may be increased (relative to Balmer lines) in (some) spaxel spectra of the MaNGA survey due to a contribution of the radiation of the diffuse ionized gas. Consequently, the abundances derived from the spaxel spectra through strong-line methods may suffer from large errors. We examined this expectation by comparing the behaviour of the line intensities and the abundances estimated through different calibrations for slit spectra of H II regions in nearby galaxies, for fibre spectra from the Sloan Digital Sky Survey, and for spaxel spectra of the MaNGA survey. We found that the $S_{2}$ strength is increased significantly in the fibre and spaxel spectra. The mean enhancement changes with metallicity and can be as large as a factor of $\sim 2$. The mean distortion of $R_{2}$ and $N_{2}$ is less than a factor of $\sim 1.3$. This suggests that Kaufmann et al.'s (2003, MNRAS, 346, 1055) demarcation line between active galactic nuclei and H II regions in the Baldwin, Phillips, \& Terlevich (BPT, 1981, PASP, 93, 5) diagram is a useful criterion to reject spectra with significantly distorted strengths of the $\mathrm{N}_{2}$ and $\mathrm{R}_{2}$ lines. We find that the three-dimensional $R$ calibration, which uses the $\mathrm{N}_{2}$ and $\mathrm{R}_{2}$ lines, produces reliable abundances in the MaNGA galaxies. The one-dimensional $\mathrm{N}_{2}$ calibration produces either reliable or wrong abundances depending on whether excitation and N/O abundance ratio in the target region (spaxel) are close to or differ from those parameters in the calibrating points located close to the calibration relation. We then determined abundance distributions within the optical radii in the discs of 47 MaNGA galaxies. The optical radii of the galaxies were estimated from the surface brightness profiles constructed based on the MaNGA observations.
\end{abstract}

Key words. galaxies: abundances - galaxies: ISM - galaxies: spiral

\section{Introduction}

It is well established that the characteristic oxygen abundance of a galaxy correlates with its macroscopic properties such as stellar galaxy mass or luminosity (Lequeux et al. 1979; Zaritsky et al. 1994; Garnett 2002; Grebel et al. 2003; Tremonti et al. 2004; Erb et al. 2006; Cowie \& Barger 2008; Maiolino et al. 2008; Guseva et al. 2009; Thuan et al. 2010; Pilyugin \& Thuan 2011; Pilyugin et al. 2013; Andrews \& Martini 2013; Zahid et al. 2013; Maier et al. 2014; Steidel et al. 2014; Izotov et al. 2015), among many authors. On the other hand, whether or not a correlation exists between the radial abundance distribution (gradient) and the macroscopic parameters of a galaxy is still not clear.

Two-dimensional (2D) spectroscopy of a large number of nearby galaxies has been carried out in the framework of surveys such as the Calar Alto Legacy Integral Field Area (CALIFA) survey (Sánchez et al. 2012a; Husemann et al. 2013; García-Benito et al. 2015) or the Mapping Nearby Galaxies at Apache Point Observatory (MaNGA) survey (Bundy et al. 2015). The 2D spectroscopy provides the possibility to determine the abundances in many regions (spaxels) across the visible surface of a galaxy and to construct an abundance map. From such data, radial abundance gradients in the discs of galaxies can be obtained. The results of these studies are somewhat contradictory.

The radial oxygen abundance distributions across the CALIFA galaxies were investigated by Sánchez et al. (2012b, 2014), and Sánchez-Menguiano et al. (2016). They found that all galaxies without clear evidence of an interaction present a common gradient in the oxygen abundance; the slope is independent of morphology, the existence of a bar, the absolute magnitude, or mass, and the distribution of the slopes is statistically compatible with a random Gaussian distribution around a mean value.

Belfiore et al. (2017) measured the oxygen abundance gradients in a sample of 550 nearby galaxies in the stellar mass range $10^{9} M_{\odot}$ to $10^{11.5} M_{\odot}$ with spectroscopic data from the SDSS-IV MaNGA survey. They found that the gradients in galaxies of $\sim 10^{9} M_{\odot}$ are roughly flat and that the metallicity gradient steepens with stellar mass until $\sim 10^{10.5} M_{\odot}$, while it becomes flatter again for higher masses.

Reliable values of the abundance gradients (and, consequently, abundances) are essential to investigate the correlation between the abundance gradient and other macroscopic 
properties of galaxies. Determination of the abundances from the spaxel spectra encounters two problems. Firstly, there is no unique metallicity scale for the $\mathrm{H}$ II regions since different methods used for the abundance determinations produce the oxygen abundances that may differ significantly. Moreover, the majority of the widely used methods do not work over the whole metallicity scale of the H II regions. Instead they are applicable for a limited range of metallicities only. This can introduce an uncertainty even in the relative values of the gradients determined with the same method in galaxies of different masses.

Secondly, it is believed that not only the H II regions but also other objects (e.g. diffuse ionized gas, DIG) can contribute to the spaxel spectra of the MaNGA survey (Belfiore et al. 2015, 2017; Zhang et al. 2017; Sanders et al. 2017). The strength of low ionization lines, [N II], [O II], and [S II], may be increased relative to Balmer lines in (some) spaxel spectra. When this happens, the abundances derived from the spaxel spectra through strong-line methods may suffer from large errors.

Before determining the abundance distributions across the discs of the MaNGA galaxies, we examine the validity of the abundances obtained through the strong-line methods from the spaxel spectra by comparing the behaviour of the line strengths and the abundances estimated through different calibrations for a sample of slit spectra of $\mathrm{H}$ II regions in nearby galaxies, of fibre spectra from the SDSS, and of spaxel spectra of the MaNGA survey. We ascertain which calibration produces the most reliable abundances from the spaxel spectra. Then the abundance distributions within the optical radii in the discs of the MaNGA galaxies are determined.

The paper is organised in the following way. The data are described in Sect. 2. In Sect. 3 the behaviour of the line intensities in slit spectra of $\mathrm{H}$ II regions in nearby galaxies, of fibre spectra from the SDSS, and of spaxel spectra of the MaNGA survey are compared and analysed. The abundances for those samples of spectra are estimated through different calibrations and examined. The abundance distributions in the discs of 47 MaNGA galaxies are obtained in Sect. 4. A discussion is given in Sect. 5 and Sect. 6 contains a brief summary.

Throughout the paper, we use the following standard notations for the line intensities:

$R_{2}=I_{[\mathrm{OII}] \lambda 3727+\lambda 3729} / I_{\mathrm{H} \beta}$,

$N_{2}=I_{[\mathrm{N} \mathrm{II}] \lambda 6548+\lambda 6584} / I_{\mathrm{H} \beta}$,

$S_{2}=I_{[\mathrm{S} \mathrm{II}]} \lambda 6717+\lambda 6731 / I_{\mathrm{H} \beta}$,

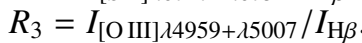

Based on these definitions, the excitation parameter $P$ is expressed as $P=R_{3} / R_{23}=R_{3} /\left(R_{2}+R_{3}\right)$. The notation $(\mathrm{O} / \mathrm{H})^{*}=$ $12+\log (\mathrm{O} / \mathrm{H})$ is used in order to permit us to write our equations in a compact way.

\section{Data for our sample of MaNGA galaxies}

The publicly available data obtained within the framework of the MaNGA SDSS DR13 survey (SDSS Collaboration 2017) form the basis of the current study. We selected our initial sample of 150 star-forming galaxies by visual examination of the images of the MaNGA galaxies with the goal being to determine the chemical (abundance distribution), kinematical (rotation curve), and photometrical (surface brightness profile) properties of these galaxies. Only galaxies for which the MaNGA observations cover the entire or a large fraction of their visible extent were selected.

For each spaxel spectrum, the fluxes of the emission lines were measured. The velocity of each region (spaxel) is estimated from the measured wavelength of the $\mathrm{H} \alpha$ line. The surface brightness in the SDSS $g$ and $r$ bands was obtained from broadband SDSS images created from the data cube. Our final sample of the MaNGA galaxies comprises 47 galaxies for which the chemical, kinematical, and photometrical properties are derived within the whole optical radius $R_{25}$ or at least up to $\sim 0.8 R_{25}$. The chemical properties of those galaxies are considered in our current study; other properties will be discussed in a forthcoming study. The MaNGA galaxies included in the final sample are listed in Table 1.

\subsection{Emission line fluxes}

The spectrum of each spaxel from the MaNGA datacubes is reduced in the manner described in Zinchenko et al. (2016). For each spectrum, the fluxes of the $[\mathrm{O}$ II $] \lambda 3727+\lambda 3729$, $\mathrm{H} \beta$, [O III] $\lambda 4959,[\mathrm{O}$ III] $\lambda 5007,[\mathrm{~N}$ II] $65548, \mathrm{H} \alpha,[\mathrm{N}$ II $] \lambda 6584$, [S II] $\lambda 6717$, and [S II] $\lambda 6731$ lines are measured. The velocity of each region (spaxel) is estimated from the measured wavelength of the $\mathrm{H} \alpha$ line. We selected spectra for which the signal-to-noise ration $(\mathrm{S} / \mathrm{N})$ is larger than three, $\mathrm{S} / \mathrm{N}>3$, for each of those lines.

The [O III] $\lambda 5007$ and $\lambda 4959$ lines originate from transitions from the same energy level, so their flux ratio is determined only by the transition probability ratio, which is very close to 3 (Storey \& Zeippen 2000). Thus, the value of $R_{3}$ can be estimated as $R_{3}=I_{[\mathrm{O} I \mathrm{II}] \lambda 4959+\lambda 5007} / I_{\mathrm{H} \beta}$ or as $R_{3}=1.33 I_{[\mathrm{OIII}] \lambda 5007} / I_{\mathrm{H} \beta}$. The stronger line $[\mathrm{O} \mathrm{III}] \lambda 5007$ is usually measured with higher precision than the weaker line [O III] $\lambda 4959$. Therefore, we estimated the value of $R_{3}$ to be $R_{3}=1.33$ [O III] $\lambda 5007 / \mathrm{H} \beta$ rather than using the sum of the line fluxes [O III] $\lambda 5007$ and $\lambda 4959$. Similarly, the $[\mathrm{N} \mathrm{II}] \lambda 6584$ and $\lambda 6548$ lines also originate from transitions from the same energy level and the transition probability ratio for those lines is again close to 3 (Storey \& Zeippen 2000). We estimated the value of $N_{2}$ to be $N_{2}=1.33$ [N II] $\lambda 6584 / \mathrm{H} \beta$ instead of the sum of the $[\mathrm{N} \mathrm{II}] \lambda 6584$ and $\lambda 6548$ lines.

The lines $[\mathrm{O} \mathrm{II}] \lambda 3727+\lambda 3729, \mathrm{H} \beta, \quad[\mathrm{O} \mathrm{III}] \lambda 5007, \mathrm{H} \alpha$, [N II] $\lambda 6584$, [S II] $\lambda 6717$, and [S II] $\lambda 6731$ are used for the dereddening and abundance determinations. Specifically, we corrected the emission-line fluxes for interstellar reddening using the theoretical $\mathrm{H} \alpha / \mathrm{H} \beta$ ratio and the Whitford (1958) interstellar reddening law (adopting the approximation suggested by Izotov et al. 1994).

We used the standard diagnostic diagram of the $[\mathrm{N}$ II $] \lambda 6584 / \mathrm{H} \alpha$ versus the $[\mathrm{O}$ III $] \lambda 5007 / \mathrm{H} \beta$ line ratios suggested by Baldwin, Phillips, Terlevich (1981), which is known as the BPT classification diagram, to separate H II region-like objects and active galactic nuclei (AGNs)-like objects. We adopted the demarcation line of Kauffmann et al. (2003) between HII regions and AGNs. The BPT diagram for our sample of the MaNGA galaxies is presented in panel a of Fig. 1. The spectra of 48591 spaxels in 47 MaNGA galaxies are used to determine the abundance distributions in the discs of those galaxies.

\subsection{Galaxy size}

\subsubsection{Preliminary remarks}

The most basic structural property of a galaxy is its size. The definition and the estimation of the galaxy size is not a trivial task. Different values (isophotal or optical radius, effective radius, Petrosian radius) have been suggested and used to specify the galaxy size.

The isophotal or optical radius is measured at or reduced to a fixed surface brightness level and corrected to a "face-on" position of a galaxy. Two values of the surface brightness level 

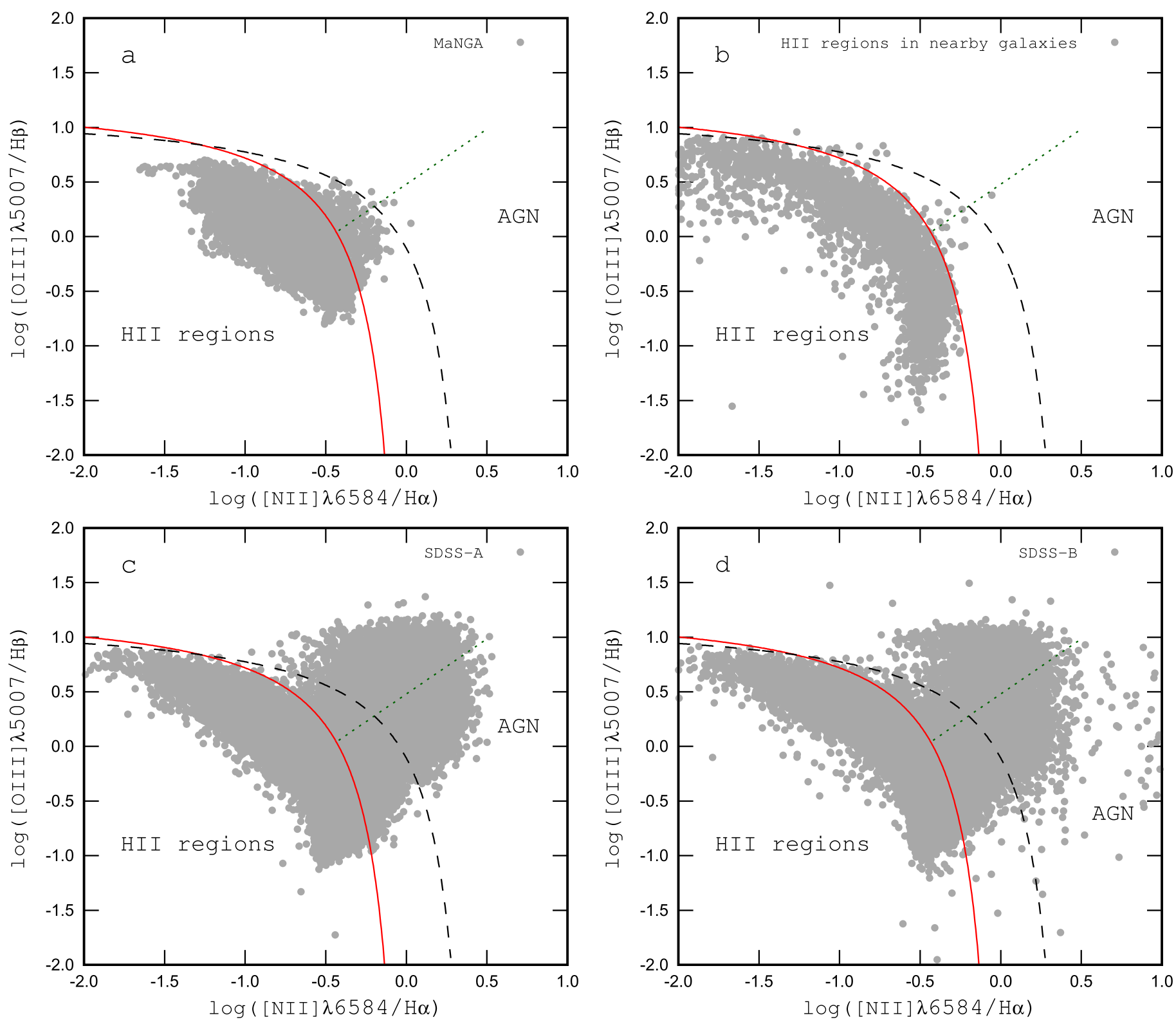

Fig. 1. Panel a: BPT diagram for our sample of MaNGA galaxies. The filled circles are individual spaxels. Solid and long-dashed curves mark the demarcation line between AGNs and H II regions defined by Kauffmann et al. (2003) and Kewley et al. (2001), respectively. The short-dashed line is the dividing line between Seyfert galaxies and LINERs defined by Cid Fernandes et al. (2010). Panel b: BPT diagram for H II regions in nearby galaxies. Panels $c$ and $d$ : BPT diagrams for fibre spectra of the SDSS galaxies with line measurements from the catalogues SDSS-A and SDSS-B, respectively.

are widely used, that is, the standard isophotal radius $R_{25}$, which refers to a level of $25.0 \mathrm{mag} / \mathrm{arcsec}^{2}$ in the $B$-band listed in the Third Reference Catalog of Bright Galaxies (de Vaucouleurs et al. 1991), and the Holmberg radius, which refers to the Holmberg isophote at a level of $26.5 \mathrm{mag} / \mathrm{arcsec}^{2}$ given in the Updated Nearby Galaxy Catalog (Karachentsev et al. 2013). Other values of the surface brightness level (and other photometric bands) are also used.

The effective radius is defined by the total magnitude of a galaxy. The total (asymptotic) magnitude of a galaxy is estimated by extrapolating the growth curve (the integrated magnitude as a function of radius) to infinity. The effective radius of a galaxy $R_{\text {eff }}$ is defined as the radius containing half of the total luminosity. The effective radius depends on the level of central concentration, in the sense that highly concentrated galaxies tend to have small values of $R_{\text {eff }}$ compared to the overall extent of the galaxy (Muñoz-Mateos et al. 2015).

The Petrosian radius is estimated through the inverse Petrosian index, which is the ratio of the surface brightness at a given radius to the average surface brightness inside this radius (Petrosian 1976). The inverse Petrosian index is estimated at each successively increasing radius. When the ratio falls below a chosen small value (usually adopted to be 0.2 ), that radius is multiplied by a factor (usually adopted to be 1.5) to obtain the Petrosian radius.

There is no one-to-one correspondence between those three values of the galaxy size. Here we use the standard isophotal radius $R_{25}$ to specify the size of the galaxy. The fractional galactocentric distances of the spaxels $R_{g}$ are normalized to the optical radius $R_{25}$. 

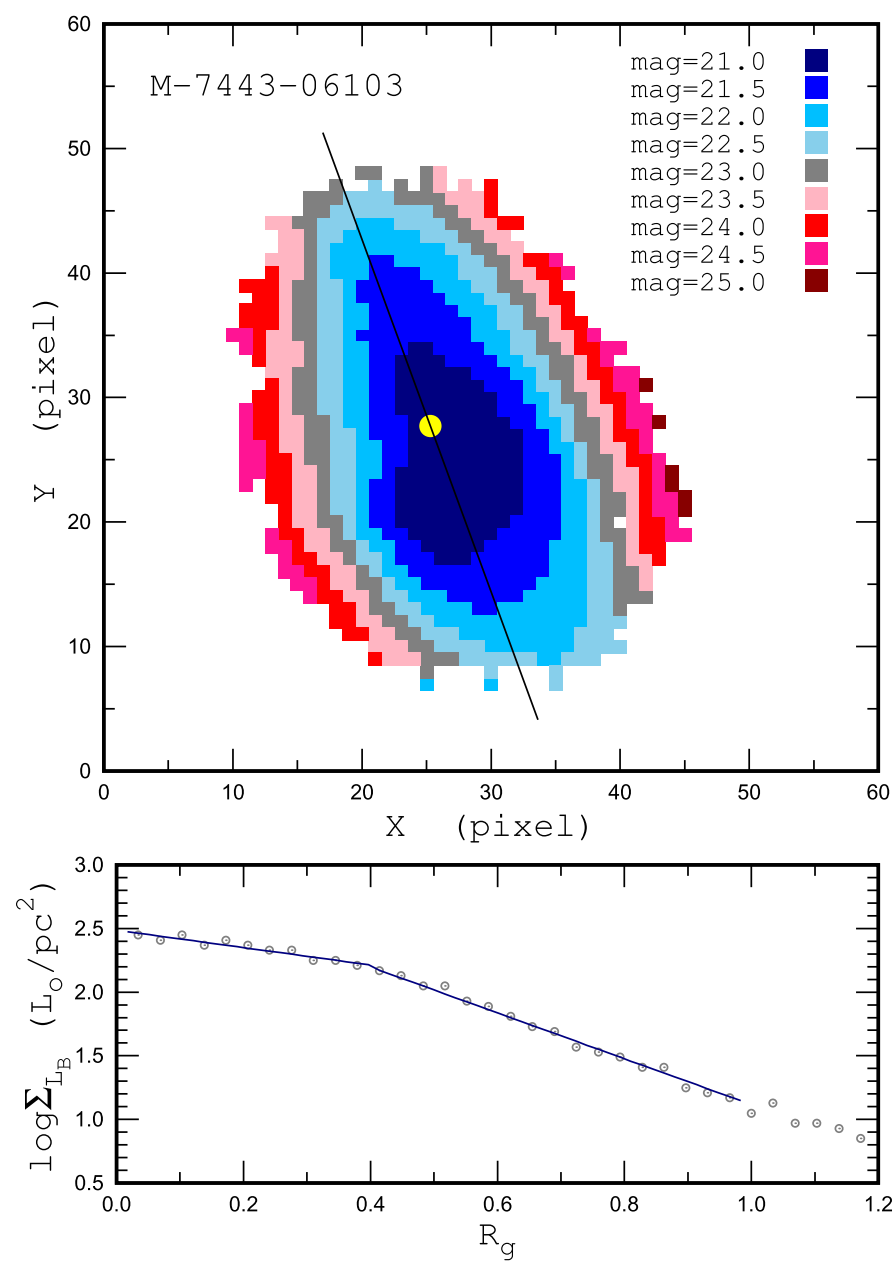

Fig. 2. Photometric properties of the MaNGA galaxy M-7443-06103. The upper panel shows the surface brightness distribution across the image of the galaxy M-7443-06103 in sky coordinates (pixels). The value of the surface brightness is colour-coded. The circle shows the kinematic centre of the galaxy. The line indicates the position of the major kinematic axis of the galaxy. The lower panel shows the observed surface-brightness profile (points) and the broken exponential fit within the isophotal radius $R_{25}$ (line).

It is interesting to note that the investigations of the extended discs of galaxies can provide a physically justified definition of the edge of a galaxy. It was found (Bresolin et al. 2009, 2012; Goddard et al. 2011; Patterson et al. 2012; Kudritzki et al. 2014; Sánchez-Menguiano et al. 2016) that the radial abundance gradient flattens to an approximately constant value outside of the isophotal radius. Bresolin et al. (2012) and Kudritzki et al. (2014) concluded that it is unlikely that the in-situ star formation outside of the isophotal radius could have produced and ejected enough metals to enrich the interstellar medium to the presently observed values. If this is a universal property of spiral galaxies then one can define the edge of a galaxy as the largest radius where the observed abundance can be produced by the stars located there.

\subsubsection{Determinations of optical radii}

The surface brightness in the SDSS $g$ and $r$ bands for each spaxel was obtained from broadband SDSS images created from the data cube. The measurements were corrected for Galactic foreground extinction using the recalibrated $A_{V}$ values of Schlafly \& Finkbeiner (2011) taken from the NASA/IPAC Extragalactic
Database (NED) ${ }^{1}$. Then the measurements in the SDSS filters $g$ and $r$ were converted to $B$-band magnitudes using the conversion relations of Blanton \& Roweis (2007),

$B_{A B}=g+0.2354+0.3915[(g-r)-0.6102]$,

where the $B_{A B}, g$, and $r$ magnitudes in Eq. (1) are in the $A B$ photometric system. The $A B$ magnitudes were reduced to the Vega photometric system

$B_{V e g a}=B_{A B}+0.09$,

which was done using the relation of Blanton \& Roweis (2007). The surface-brightness profile was determined from the surface brightness map with the galaxy inclination and the position angle of the major axis obtained through the analysis of the observed velocity field. Those values will be discussed in a forthcoming paper. The upper panel of Fig. 2 shows the surface-brightness distribution across the image of the galaxy M-7443-06103 in sky coordinates (pixels). The position of the kinematic centre of the galaxy is marked by the circle and the major kinematic axis of the galaxy is indicated by the line in the upper panel of Fig. 2. The lower panel shows the observed surface-brightness profile (points) for the galaxy M-7443-06103.

We use the surface-brightness profile in solar units for the bulge-disc decomposition. The magnitude of the Sun in the $B$ band of the Vega photometric system, $B_{\odot}=5.45$, was taken from Blanton \& Roweis (2007). The distances were adopted from the NED. The NED distances use flow corrections for Virgo, the Great Attractor, and Shapley Supercluster infall. The stellar surface-brightness distribution within a galaxy was fitted by a broken exponential profile for the disc and by a general Sérsic profile for the bulge.

The total surface-brightness distribution was fitted with the expression

$$
\begin{aligned}
\Sigma_{L}(r)= & \left(\Sigma_{L}\right)_{e} \exp \left\{-b_{n}\left[\left(r / r_{e}\right)^{1 / n}-1\right]\right\} \\
& +\left(\Sigma_{L}\right)_{0, \text { inner }} \exp \left(-r / h_{\text {inner }}\right) \quad \text { if } \quad r<R^{*} \\
= & \left(\Sigma_{L}\right)_{e} \exp \left\{-b_{n}\left[\left(r / r_{e}\right)^{1 / n}-1\right]\right\} \\
& +\left(\Sigma_{L}\right)_{0, \text { outer }} \exp \left(-r / h_{\text {outer }}\right) \quad \text { if } \quad r>R^{*}
\end{aligned}
$$

Here $R^{*}$ is the break radius, that is, the radius at which the exponent changes. The eight parameters $\left(\Sigma_{L}\right)_{e}, r_{e}, n,\left(\Sigma_{L}\right)_{0, \text { inner }}$, $h_{\text {inner }},\left(\Sigma_{L}\right)_{0, \text { outer }}, h_{\text {outer }}$, and $R^{*}$ in the broken exponential disc were determined through the best fit to the observed surfacebrightness profile, that is, we require that the deviation $\sigma_{B E D}$, given by

$\sigma=\sqrt{\left[\sum_{j=1}^{n}\left(L\left(r_{j}\right)^{c a l} / L\left(r_{j}\right)^{o b s}-1\right)^{2}\right] / n}$,

be minimized. Here $L\left(r_{j}\right)^{c a l}$ is the surface brightness at the radius $r_{j}$ computed through Eq. (3) and $L\left(r_{j}\right)^{o b s}$ is the measured surface brightness at that radius. The broken exponential fit to the surface-brightness profile of the galaxy M-7443-06103 is shown by the line in the lower panel of Fig. 2.

The isophotal radius of the galaxy, the central surface brightness of the disc, and the galaxy luminosity estimated using Eq. (3) are reported in Table 1.

1 The NASA/IPAC Extragalactic Database (NED) is operated by the Jet Populsion Laboratory, California Institute of Technology, under contract with the National Aeronautics and Space Administration. http://ned.ipac.caltech.edu/ 
Table 1. Characteristics of MaNGA galaxies of our sample.

\begin{tabular}{|c|c|c|c|c|c|c|c|c|c|c|}
\hline Name & $\underset{\circ}{\mathrm{RA}}$ & $\underset{\circ}{\operatorname{Dec}}$ & $\begin{array}{c}d \\
\mathrm{Mpc}\end{array}$ & $\begin{array}{c}R_{25} \\
\operatorname{arcmin} \\
\end{array}$ & $\begin{array}{l}R_{25} \\
\mathrm{kpc} \\
\end{array}$ & $\begin{array}{c}\log L_{B} \\
L_{\odot} \\
\end{array}$ & $\begin{array}{l}\log \Sigma_{0} \\
L_{\odot} / \mathrm{pc}^{2} \\
\end{array}$ & $12+\log (\mathrm{O} / \mathrm{H})_{0}$ & $\begin{array}{c}\text { Gradient } \mathrm{O} / \mathrm{H} \\
\mathrm{dex} / \mathrm{R}_{25}\end{array}$ & $\begin{array}{c}\sigma \mathrm{O} / \mathrm{H} \\
\operatorname{dex}\end{array}$ \\
\hline 744306103 & 230.233668 & 42.775541 & 82.8 & 0.24 & 5.82 & 9.61 & 2.20 & 8.41 & -0.081 & 0.0547 \\
\hline 795706104 & 258.220415 & 35.093157 & 111.2 & 0.27 & 8.63 & 10.00 & 2.31 & 8.47 & -0.173 & 0.0535 \\
\hline 797706103 & 331.802634 & 13.266052 & 143.5 & 0.16 & 6.61 & 9.34 & 1.69 & 8.53 & -0.325 & 0.0554 \\
\hline 813112701 & 111.109940 & 38.945215 & 72.5 & 0.36 & 7.56 & 9.56 & 2.04 & 8.46 & -0.379 & 0.0680 \\
\hline 813412701 & 112.698680 & 46.010115 & 88.9 & 0.32 & 8.19 & 9.51 & 1.84 & 8.54 & -0.309 & 0.0669 \\
\hline 813412703 & 114.473654 & 47.957220 & 88.1 & 0.30 & 7.69 & 9.42 & 1.71 & 8.52 & -0.231 & 0.0708 \\
\hline 813412705 & 114.673017 & 45.943654 & 76.4 & 0.33 & 7.41 & 9.41 & 1.77 & 8.59 & -0.235 & 0.0552 \\
\hline 813503704 & 114.897365 & 37.751505 & 126.7 & 0.28 & 10.13 & 10.06 & 2.58 & 8.48 & -0.228 & 0.0564 \\
\hline 813806104 & 117.291419 & 46.461883 & 158.4 & 0.15 & 6.91 & 9.49 & 1.77 & 8.47 & -0.175 & 0.0579 \\
\hline 814009101 & 116.507218 & 40.875058 & 157.9 & 0.17 & 8.04 & 9.66 & 2.06 & 8.43 & -0.245 & 0.0696 \\
\hline 814306102 & 118.946946 & 41.280373 & 170.1 & 0.21 & 10.31 & 9.79 & 1.93 & 8.46 & -0.275 & 0.0711 \\
\hline 824309101 & 128.178383 & 52.416775 & 179.8 & 0.27 & 13.95 & 10.22 & 2.34 & 8.44 & -0.208 & 0.0542 \\
\hline 824712701 & 138.275133 & 43.013463 & 62.1 & 0.31 & 5.57 & 9.11 & 1.63 & 8.24 & -0.190 & 0.0664 \\
\hline 825012702 & 140.359627 & 43.102154 & 188.9 & 0.22 & 11.91 & 9.91 & 1.96 & 8.62 & -0.327 & 0.0494 \\
\hline 825012703 & 139.647743 & 44.596737 & 62.9 & 0.28 & 5.03 & 8.96 & 1.46 & 8.25 & -0.216 & 0.0798 \\
\hline 825212703 & 145.456567 & 48.648991 & 189.8 & 0.20 & 11.04 & 9.87 & 2.10 & 8.64 & -0.247 & 0.0478 \\
\hline 825303703 & 157.343576 & 43.170589 & 116.7 & 0.20 & 6.79 & 9.88 & 2.60 & 8.59 & -0.019 & 0.0181 \\
\hline 825309102 & 158.207632 & 43.173946 & 124.4 & 0.38 & 13.57 & 10.11 & 2.17 & 8.52 & -0.309 & 0.0456 \\
\hline 825703704 & 165.553613 & 45.303871 & 88.8 & 0.24 & 6.24 & 9.52 & 2.04 & 8.27 & -0.179 & 0.0647 \\
\hline 825803701 & 165.570030 & 44.335183 & 106.4 & 0.19 & 5.93 & 9.71 & 2.86 & 8.63 & -0.310 & 0.0458 \\
\hline 825806101 & 168.015359 & 42.669794 & 93.3 & 0.25 & 6.78 & 9.37 & 1.80 & 8.27 & -0.143 & 0.0618 \\
\hline 825912701 & 179.962635 & 43.740689 & 87.1 & 0.32 & 8.02 & 9.48 & 1.71 & 8.32 & -0.194 & 0.0657 \\
\hline 825912702 & 178.506318 & 44.642256 & 104.5 & 0.38 & 11.40 & 9.85 & 1.87 & 8.62 & -0.256 & 0.0435 \\
\hline 831712705 & 193.855952 & 43.938063 & 165.3 & 0.21 & 10.02 & 9.61 & 1.64 & 8.50 & -0.216 & 0.0739 \\
\hline 831812705 & 197.411496 & 45.269246 & 125.8 & 0.33 & 11.89 & 10.15 & 2.22 & 8.65 & -0.366 & 0.0410 \\
\hline 832012701 & 205.548609 & 22.269154 & 180.3 & 0.19 & 10.05 & 9.70 & 2.00 & 8.52 & -0.246 & 0.0593 \\
\hline 832012703 & 206.630975 & 23.122136 & 132.7 & 0.26 & 9.97 & 9.66 & 1.72 & 8.53 & -0.272 & 0.0473 \\
\hline 832512701 & 210.176336 & 45.833422 & 182.9 & 0.26 & 13.74 & 9.88 & 1.77 & 8.57 & -0.256 & 0.0631 \\
\hline 832512705 & 212.101063 & 47.273720 & 168.4 & 0.21 & 10.21 & 9.76 & 1.88 & 8.61 & -0.306 & 0.0507 \\
\hline 832912704 & 213.783652 & 45.594849 & 72.9 & 0.42 & 9.01 & 9.86 & 2.23 & 8.47 & -0.304 & 0.0564 \\
\hline 833506102 & 216.904792 & 40.407305 & 86.8 & 0.28 & 7.15 & 9.59 & 2.40 & 8.42 & -0.218 & 0.0606 \\
\hline 834109102 & 191.012464 & 45.129265 & 107.4 & 0.24 & 7.55 & 9.68 & 1.99 & 8.58 & -0.146 & 0.0394 \\
\hline 843906101 & 143.184618 & 48.796348 & 111.9 & 0.25 & 8.14 & 9.76 & 2.17 & 8.57 & -0.265 & 0.0511 \\
\hline 843909101 & 142.713904 & 50.318861 & 159.0 & 0.13 & 6.17 & 9.37 & 1.79 & 8.49 & -0.220 & 0.0537 \\
\hline 843912701 & 143.010196 & 48.551093 & 71.4 & 0.37 & 7.62 & 9.26 & 1.61 & 8.43 & -0.257 & 0.0740 \\
\hline 844012703 & 136.748725 & 41.440882 & 110.8 & 0.27 & 8.59 & 9.65 & 1.90 & 8.51 & -0.311 & 0.0546 \\
\hline 844712703 & 207.483361 & 40.895076 & 150.8 & 0.12 & 5.12 & 8.89 & 1.26 & 8.47 & -0.219 & 0.0738 \\
\hline 845112703 & 164.028900 & 43.156568 & 160.4 & 0.25 & 11.66 & 9.86 & 1.99 & 8.41 & -0.187 & 0.0742 \\
\hline 845312702 & 151.547771 & 47.295038 & 160.7 & 0.21 & 9.74 & 9.56 & 1.51 & 8.53 & -0.216 & 0.0665 \\
\hline 845906102 & 147.990674 & 43.414043 & 70.1 & 0.23 & 4.59 & 9.20 & 2.13 & 8.33 & -0.171 & 0.0544 \\
\hline 845912704 & 147.604836 & 44.040637 & 70.0 & 0.23 & 4.75 & 8.92 & 1.59 & 8.48 & -0.232 & 0.0651 \\
\hline 845912705 & 148.117076 & 42.819141 & 71.9 & 0.38 & 7.84 & 9.48 & 1.71 & 8.52 & -0.294 & 0.0595 \\
\hline 846609101 & 170.214847 & 44.597411 & 106.8 & 0.27 & 8.28 & 9.57 & 1.74 & 8.56 & -0.289 & 0.0591 \\
\hline 846609102 & 170.583304 & 46.701332 & 77.8 & 0.28 & 6.41 & 9.53 & 2.07 & 8.28 & -0.210 & 0.0565 \\
\hline 848512701 & 233.319217 & 48.119650 & 102.7 & 0.28 & 8.22 & 9.52 & 1.82 & 8.34 & -0.189 & 0.0602 \\
\hline 854806103 & 243.472257 & 48.296700 & 89.9 & 0.33 & 8.72 & 9.82 & 2.26 & 8.45 & -0.255 & 0.0396 \\
\hline 854906103 & 240.418740 & 46.085291 & 177.2 & 0.23 & 11.60 & 9.82 & 1.91 & 8.43 & -0.200 & 0.0560 \\
\hline
\end{tabular}

Notes. The first column gives the galaxy's identification number in the MaNGA survey. The right ascension (RA) and declination (Dec) (J2000.0) of each galaxy are given in columns 2 and 3. The adopted distance is reported in column 4 . The isophotal radius in arcmin and in kpc are listed in columns 5 and 6 , respectively. The luminosity and the central surface brightness of the disc (reduced to the face-on) in the $B$ band estimated here are given in columns 7 and 8 . The central oxygen abundance and the radial abundance gradient estimated using the $R$ calibration are reported in columns 9 and 10 . Column 11 gives the value of the mean scatter around the $\mathrm{O} / \mathrm{H}-R_{g}$ relation.

It should be noted that the bulge contribution is small in each of the galaxies considered, and the stellar surfacebrightness distribution within a galaxy is well-fitted by a broken exponential profile for the disc, that is, our sample of MaNGA galaxies can be considered as a sample of bulgeless galaxies. 


\subsection{Abundances}

\subsubsection{Calibrations used}

It is believed that the radiation of the DIG can contribute significantly to (some) spaxel spectra of the MaNGA survey, and the strength of the low-ionization lines [N II], [O II], and [S II] can be increased relative to the Balmer lines. As a result, the abundances derived from the spaxel spectra through strong-line methods may have large errors (Belfiore et al. 2015, 2017; Zhang et al. 2017; Sanders et al. 2017). We consider four calibrations based on different low-ionization emission lines to estimate the oxygen abundances from the spaxel spectra. On the one hand, the use of the calibrations based on different emission lines allows us to draw conclusions about the validity of the abundances and to choose the calibration that produces the most reliable abundances. On the other hand, this provides the possibility to establish which of the low-ionization emission lines are significantly distorted by the contribution of the radiation of the DIG to the spaxel spectra.

We use the simple three-dimensional (3D) calibration relations for abundance determinations from a set of strong emission lines suggested in Pilyugin \& Grebel (2016). The oxygen abundances $(\mathrm{O} / \mathrm{H})_{R}$ are determined using the oxygen $\mathrm{R}_{2}, \mathrm{R}_{3}$ lines and the nitrogen $\mathrm{N}_{2}$ line ( $R$ calibration). The oxygen $\mathrm{R}_{3}$ line, the nitrogen $\mathrm{N}_{2}$ line, and the sulphur $\mathrm{S}_{2}$ line (instead of the oxygen $\mathrm{R}_{2}$ line) are used in the determinations of the $(\mathrm{O} / \mathrm{H})_{S}$ abundances through the $S$ calibration introduced in Pilyugin \& Grebel (2016).

The 2D $P$ calibration is based on the oxygen $\mathrm{R}_{2}$ and $\mathrm{R}_{3}$ lines only (Pilyugin 2000, 2001; Pilyugin \& Thuan 2005). It is important that the calibration relations were consistent with each other. We obtained the $P$ calibration relation for high-metallicity objects, $12+\log (\mathrm{O} / \mathrm{H}) \gtrsim 8.35$, (the so-called upper-branch calibration) using the $(\mathrm{O} / \mathrm{H})_{R}$ abundances for the calibrating data points (the compilation of spectra of $\mathrm{H}$ II regions in nearby galaxies) from Pilyugin et al. (2012, 2014), and Pilyugin \& Grebel (2016)). The obtained upper-branch $P$ calibration relation is

$$
\begin{aligned}
(\mathrm{O} / \mathrm{H})_{\mathrm{P}}^{*}= & 7.852+0.332 P \\
& +(-0.560-0.176 P) \times\left(\log R_{3}-1\right) \\
& +(-0.120+0.109 P) \times\left(\log R_{3}-1\right)^{2}
\end{aligned}
$$

where $P$ is the excitation parameter. The notation $(\mathrm{O} / \mathrm{H})_{P}^{*}=$ $12+\log (\mathrm{O} / \mathrm{H})_{P}$ is used for the sake of brevity.

The nitrogen $\mathrm{N}_{2}$ line is the basis of the one-dimensional (1D) $\mathrm{N}_{2}$ calibration of Pettini \& Pagel (2004). We obtained the calibration relation for objects with $\log \mathrm{N}_{2}>-0.6$ using again the $(\mathrm{O} / \mathrm{H})_{R}$ abundances for the calibrating data points

$$
12+\log (\mathrm{O} / \mathrm{H})_{N}=8.485+0.616 \log \mathrm{N}_{2} .
$$

It should be noted that the nitrogen $\mathrm{N}_{2}$ line is used in this relation but not the $\mathrm{N}_{2}$ abundance indicator of (Pettini \& Pagel 2004; see below).

Thus, we use four calibrations based on emission lines of different low-ionization ions. Only the nitrogen line $\mathrm{N}_{2}$ is used in the determinations of the $(\mathrm{O} / \mathrm{H})_{N}$ abundances, only the oxygen line $\mathrm{R}_{2}$ is used in the determinations of the $(\mathrm{O} / \mathrm{H})_{P}$ abundances, the oxygen $\mathrm{R}_{2}$ and nitrogen $\mathrm{N}_{2}$ lines are used in the determinations of the $(\mathrm{O} / \mathrm{H})_{R}$ abundances, and the nitrogen $\mathrm{N}_{2}$ and sulphur $\mathrm{S}_{2}$ lines are used in the determinations of the $(\mathrm{O} / \mathrm{H})_{S}$ abundances.

\subsubsection{Abundance gradients}

The radial oxygen abundance distribution in a galaxy is traditionally described by the linear relation

$$
12+\log (\mathrm{O} / \mathrm{H})(R)=12+\log (\mathrm{O} / \mathrm{H})_{0}+\operatorname{grad} \times R,
$$

where $12+\log (\mathrm{O} / \mathrm{H})(R)$ is the oxygen abundance at the galactocentric distance $R$ in $\mathrm{kpc}, 12+\log (\mathrm{O} / \mathrm{H})_{0}$ is the extrapolated central oxygen abundance, and grad is the slope of the oxygenabundance gradient expressed in terms of dex/kpc. The linear relation is a rather good approximation for the abundance distributions in the discs of spiral galaxies (e.g. Pilyugin et al. 2017, and discussion there). This is a standard physical scale where the value of the gradient specifies the change of the abundance in the disc as a function of the change of the radius in kiloparsecs.

There is also another widely used definition of the gradient where the change of the abundance refers not to the kpc scale but to some fixed scale of galactocentric distance. The gradient is often defined as the change of the abundance in the disc over its isophotal radius $R_{25}$ and is expressed in terms of dex/R $R_{25}$. In this case, the galactocentric distance is normalized to the disc's isophotal radius, $R_{g}=R / R_{25}$. With this definition, the value of the gradient means the difference between the abundance at the optical radius of a galaxy and the central abundance.

Moreover, in a number of investigations, the gradient is defined as the change of the abundance in the disc as a function of its effective radius and is expressed in terms of dex/ $R_{\text {eff }}$. In this case, the galactocentric distance is normalized to the disc's effective radius, $R^{*}=R / R_{\text {eff }}$.

It is evident that

$\operatorname{grad}_{R_{\text {scale }}}=R_{\text {scale }} \times \operatorname{grad}_{\mathrm{kpc}}$,

where $R_{\text {scale }}=R_{25}$ or $R_{\text {scale }}=R_{\text {eff }} . R_{\text {scale }}=1$ for the canonical definition of the gradient.

We will use below the definition of the gradient where the change of the abundance refers to the isophotal radius $R_{25}$ of a galaxy and is expressed in terms of dex/R $R_{25}$. The radial oxygen abundance distribution in each galaxy of our sample is fitted by a linear relation. If there were points that showed large deviations from the $\mathrm{O} / \mathrm{H}-R_{g}$ relation $\left(d_{\mathrm{OH}}>0.2 \mathrm{dex}\right)$ then those points were not used in deriving the final relations and were excluded from further analysis. It should be noted that points with such large deviations are present only in a few galaxies and those points are not very numerous. The mean deviation from the final relations (the mean value of the residuals of the relations) is given by

$\sigma_{\mathrm{OH}}=\left(\frac{1}{n} \sum_{j=1}^{n}\left(\log (\mathrm{O} / \mathrm{H})_{j}^{\mathrm{OBS}}-\log (\mathrm{O} / \mathrm{H})_{j}^{\mathrm{CAL}}\right)^{2}\right)^{1 / 2}$,

and is usually around 0.04 dex to $0.07 \mathrm{dex}$; see Table 1 . Thus, the deviations of the rejected points from the $\mathrm{O} / \mathrm{H}-R_{g}$ relation exceed $3-5 \sigma_{\mathrm{OH}}$.

\section{Abundances derived from $\mathrm{H}_{\mathrm{II}}$ region slit spectra, from SDSS fibre spectra, and from MaNGA spaxel spectra}

It is currently believed that the radiation of DIG can make a significant contribution to (some) observed fibre spectra of the SDSS survey and (some) spaxel spectra of the MaNGA survey and that the strength of the low-ionization lines, [N II], [O II], and 

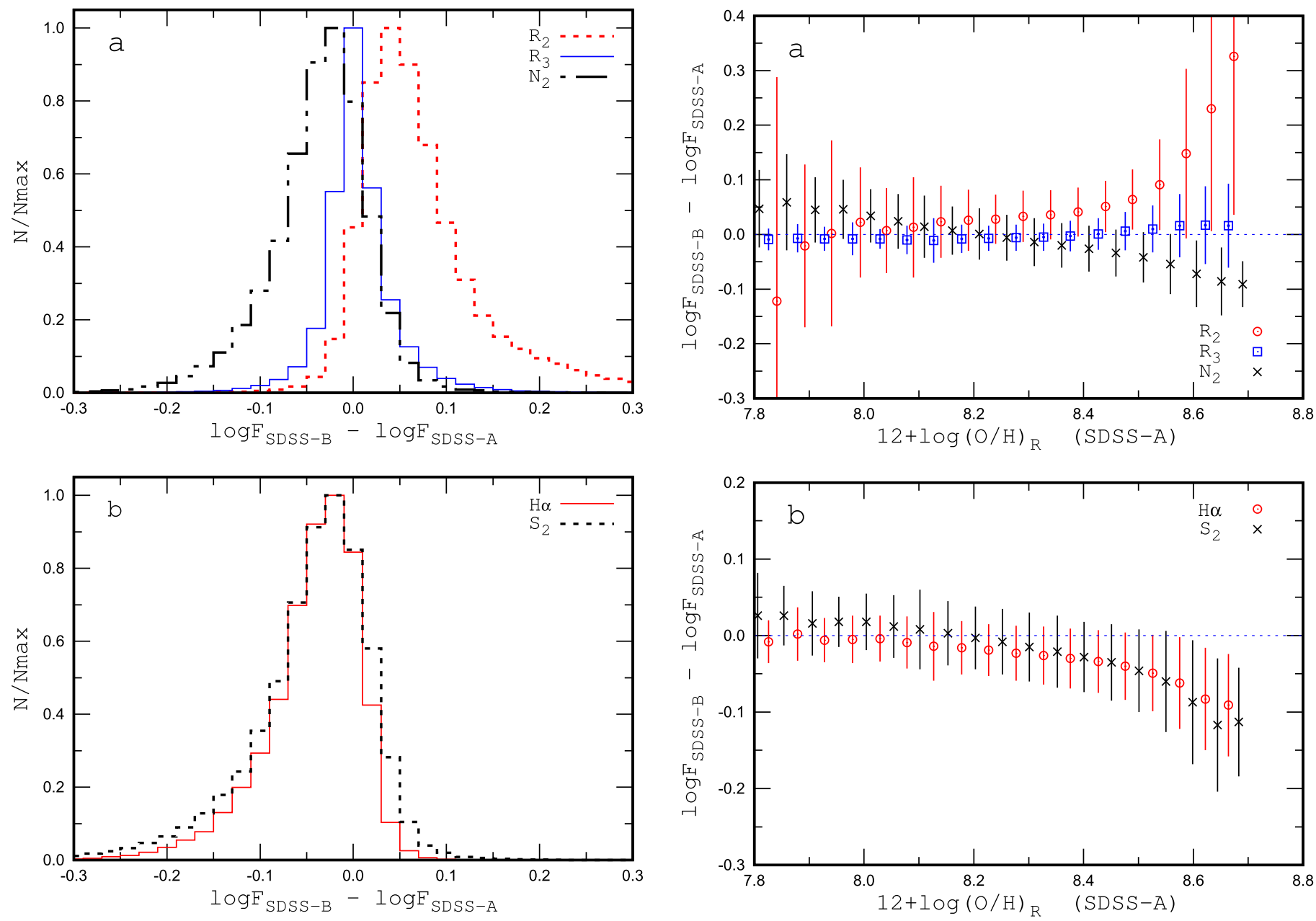

Fig. 3. Normalized histograms of the differences between the measured (non-dereddened) emission-line intensities in the SDSS-A and SDSS-B catalogues of the SDSS fibre spectra measurements.

[S II], can be increased relative to the Balmer lines (e.g. Tremonti et al. 2004; Belfiore et al. 2015, 2017; Zhang et al. 2017; Sanders et al. 2017).

Therefore, the abundances derived using strong-line methods from spectra contaminated by the radiation of diffuse ionized gas can show large errors. Zhang et al. (2017) found that the use of some calibrations to derive metallicities introduce a bias in the derived gradients as large as the gradient itself. We have examined the abundances estimated from slit spectra of a large sample of H II regions in nearby galaxies, from fibre spectra of SDSS galaxies, and from spaxel spectra of MaNGA galaxies. The abundances were determined through different calibrations (based on different emission lines). If the radiation of the diffuse ionized gas produces a distortion of the different low-ionization emission lines in the MaNGA spaxel spectra (e.g. Sanders et al. 2017) then the abundances determined using those lines as the abundance indicator will be distorted to different extents. The comparison of abundances determined through different calibrations allows us to estimate the influence of the diffuse ionized gas on these determinations and to choose the calibration(s) producing the most reliable abundances from the spaxel spectra of the MaNGA galaxies.

\subsection{Samples considered}

In our previous studies we compiled a large number of slit spectra of $\mathrm{H}$ II regions in nearby spiral and irregular galaxies

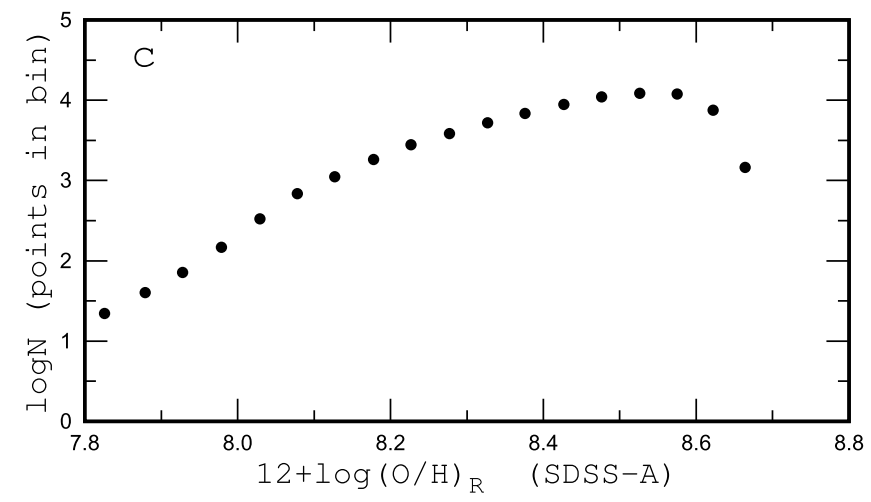

Fig. 4. Panels $a$ and $b$ : differences between the measured (nondereddened) emission-line intensities in the SDSS-A and SDSS-B catalogues. The symbols represent the mean values of the differences for objects in bins of 0.05 dex in $(\mathrm{O} / \mathrm{H})_{R, S D S S-A}$. The bars denote the mean values of the scatter of the differences for objects in bins. Panel $c$ : number of points (objects) in the bin.

(Pilyugin et al. 2012, 2014; Pilyugin \& Grebel 2016). The BPT diagram for our sample of $\mathrm{H}$ II regions is presented in panel b of Fig. 1. Some spectra located to the right of (or above) the demarcation line of Kauffmann et al. (2003) were rejected. The final sample of the $\mathrm{H}$ II regions includes 3440 spectra with measured $\mathrm{R}_{2}, \mathrm{R}_{3}, \mathrm{~N}_{2}$, and $\mathrm{S}_{2}$ lines.

Two samples of emission-line fluxes in the SDSS fibre spectra are considered. Firstly, the emission-line flux measurements in the SDSS fibre spectra were taken from the MPA/JHU catalogue (galSpecLine, SDSS-III DR12) of automatic line flux 


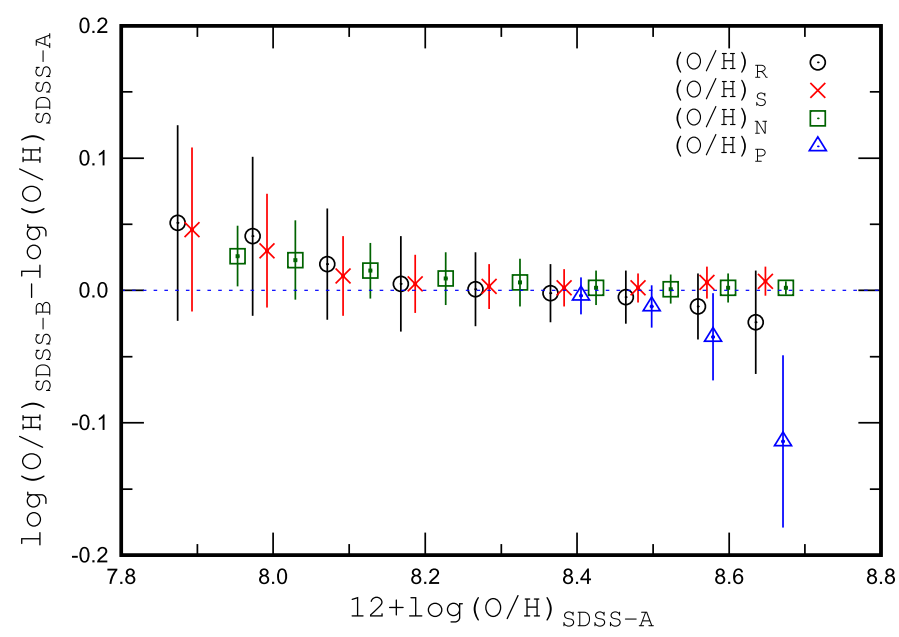

Fig. 5. Comparison of the oxygen abundances of the SDSS objects determined through the different calibrations using the emission-line flux measurements from the SDSS-A and SDSS-B catalogues. The symbols represent the mean values of the abundance differences for objects in bins of 0.1 dex in $(\mathrm{O} / \mathrm{H})_{S D S S-A}$. The bars denote the mean values of the scatter of the abundance differences for objects in the bins.

measurements of the SDSS spectra (see Brinchmann et al. (2004); Tremonti et al. (2004) and other publications of those authors). The stellar population models from Bruzual \& Charlot (2003) are used for the continuum fitting in this catalogue. We select from the catalogue those objects for which the $\mathrm{S} / \mathrm{N}$ is larger than 3 for each of the $\mathrm{H}_{\beta}, \mathrm{H}_{\alpha}, \mathrm{R}_{2}, \mathrm{R}_{3}, \mathrm{~N}_{2}$, and $\mathrm{S}_{2}$ lines in the spectra, and that are located left of (below) the demarcation line of Kauffmann et al. (2003) in the BPT diagram in panel c of Fig. 1. These criteria yield a sample of 193.037 SDSS objects, which we call the SDSS-A sample.

Secondly, we also used emission-line flux measurements for the SDSS fibre spectra from the catalogue (emissionLinesPort, SDSS-III DR12). The stellar population models from Maraston \& Strömbäck (2011) and Thomas et al. (2011) are used for the continuum fitting in this catalogue. The same criteria as for the SDSS-A sample are used to select the objects from this catalogue. This sample involves 87.381 SDSS objects and is called SDSS-B sample below. The BPT diagram for SDSS-B sample is presented in panel d of Fig. 1.

The MaNGA sample includes our measurements of the 48.591 spaxel spectra in 47 MaNGA galaxies (see above).

\subsection{Uncertainty in the SDSS-fibre-based abundances due to the uncertainty in the data reduction and emission-line measurements}

The samples SDSS-A and SDSS-B have 75.967 objects in common. This provides the possibility to assess the uncertainties of the data reduction and emission-line flux measurements in the SDSS fibre spectra and corresponding uncertainties in the abundances determined through the different calibrations.

Figure 3 shows the normalized histograms of the differences of the measured (non-dereddened) emission-line intensities in the SDSS-A and SDSS-B catalogues of the SDSS fibre spectra. Inspection of Fig. 3 reveals systematic differences between the emission-line intensities in the SDSS-A and SDSS-B catalogues. The difference is dependent on the wavelength of the emission line, in the sense that the measured intensities of the ultraviolet line $\mathrm{R}_{2}$ are stronger in the SDSS-B catalogue than in the
SDSS-A catalogue while the red lines, $\mathrm{N}_{2}, \mathrm{H} \alpha$, and $\mathrm{N}_{2}$, are stronger in the SDSS-A catalogue than in the SDSS-B catalogue.

Panels a and b of Fig. 4 show the differences of the measured emission-line intensities between the SDSS-A and SDSS-B catalogues of the SDSS fibre spectra as a function of oxygen abundance $(\mathrm{O} / \mathrm{H})_{R}$ estimated from the line measurements from the SDSS-A catalogue. The symbols represent the mean values of the differences for objects in bins of $0.05 \mathrm{dex}$ in $(\mathrm{O} / \mathrm{H})_{R}$. The bars denote the mean values of the scatter of the differences in the bins. Panel c shows the number of points (objects) in the bins. Figure 4 shows that the difference between the measured emission-line intensities in the SDSS-A and SDSS-B catalogues changes with oxygen abundance. The $\mathrm{R}_{3}$ line intensities are rather close in both catalogues. The $\mathrm{N}_{2}, \mathrm{H} \alpha$, and $\mathrm{S}_{2}$ line intensities are higher in the SDSS-B catalogue at low metallicities and the differences $\mathrm{F}_{S D S S-B}-\mathrm{F}_{S D S S-A}$ decrease with metallicity and reach zero at the metallicity $12+\log (\mathrm{O} / \mathrm{H}) \sim 8.25$. The intensities of those lines in the SDSS-B catalogue are lower, on average, than in the SDSS-A catalogue at high metallicities, $12+\log (\mathrm{O} / \mathrm{H}) \gtrsim 8.25$. The absolute difference increases with abundance and becomes as large as $\sim 0.1 \mathrm{dex}$ at metallicities of $12+\log (\mathrm{O} / \mathrm{H}) \gtrsim$ 8.6. The intensity of the $\mathrm{R}_{2}$ line is larger in the SDSS-B catalogue than in the SDSS-A catalogue at any metallicity and the difference exceeds 0.1 dex at $12+\log (\mathrm{O} / \mathrm{H})$ $\gtrsim 8.6$.

Figure 5 shows the comparison of the oxygen abundances determined through the different calibrations using the emissionline measurements from the SDSS-A and SDSS-B catalogues. The symbols represent the mean values of the abundance differences for objects in bins of $0.1 \mathrm{dex}$ in $(\mathrm{O} / \mathrm{H})_{S D S S-A}$. The centres of the bins for abundances based on different calibrations are shifted in order to avoid the coincidence of the positions of the symbols in the figure. The bars denote the mean values of the scatter of the abundance differences in the bins.

Figure 5 shows that the $(\mathrm{O} / \mathrm{H})_{R}$ (as well as $(\mathrm{O} / \mathrm{H})_{S}$ and $\left.(\mathrm{O} / \mathrm{H})_{N}\right)$ abundances determined using the emission-line measurements from the SDSS-A and SDSS-B catalogues agree within around 0.02 dex for the objects with metallicities $12+$ $\log (\mathrm{O} / \mathrm{H}) \gtrsim 8.1$. The difference is larger at lower metallicities and is $\sim 0.05$ dex at $12+\log (\mathrm{O} / \mathrm{H}) \lesssim 8.0$. The difference between the $(\mathrm{O} / \mathrm{H})_{P}$ abundances determined using the emission-line measurements from the SDSS-A and SDSS-B catalogues is large for the objects with metallicities $12+\log (\mathrm{O} / \mathrm{H}) \gtrsim 8.55$. Here the absolute value of the difference exceeds 0.1 dex.

Thus, the uncertainties in abundances estimated through the different calibrations due to the uncertainties in the data reduction and line flux measurements are within $\sim 0.02$ dex for objects with metallicities from $12+\log (\mathrm{O} / \mathrm{H}) \sim 8.1$ to $12+\log (\mathrm{O} / \mathrm{H}) \sim$ 8.55 and are higher at lower and higher metallicities.

It may appear surprising that considerably different line intensities in the SDSS-A and SDSS-B catalogues (Fig. 4) yield very similar abundances (Fig. 5). This can be explained by the following. Since the $\mathrm{H} \alpha / \mathrm{H} \beta$ ratio is higher in the SDSS-A catalogue and, consequently, the extinction parameter $C_{\mathrm{H} \beta}$ is also higher, the dereddening results in a decrease of the systematic difference in the measured line intensities between the SDSS-A and SDSS-B catalogues. We have also dereddened the SDSS-A and SDSS-B spectra using the reddening function from Cardelli et al. (1989) for $R_{V}=3.1$ to check the robustness of the results. We assume $C_{\mathrm{H} \beta}=0.47 A_{V}$ following Lee et al. (2005). There is no significant difference between the results obtained with Whitford's and Cardelli et al.'s reddening laws.

It is thus difficult to choose which catalogue (SDSS-A or SDSS-B) is more reliable. 


\subsection{Abundances determined from $\mathrm{H}_{\mathrm{II}}$ region slit spectra, from SDSS fibre spectra, and from MaNGA spaxel spectra}

Here we compare the abundances determined through different calibrations from the $\mathrm{H}$ II region slit spectra, from the SDSS fibre spectra, and from the MaNGA spaxel spectra.

\subsubsection{Line ratios}

Firstly we compare the line intensities in the spectra of our samples. The objects of each sample are binned in the $\mathrm{N}_{2}$ line intensities. The $\mathrm{N}_{2}$ value is an indicator of the electron temperature and abundance in a H II region; for an example, see Fig. 2 in Pilyugin \& Grebel (2016). Thus the binning in the $\mathrm{N}_{2}$ values can be considered as a binning in abundances. Furthermore, the binned $\mathrm{N}_{2}, \mathrm{R}_{2}$, and $\mathrm{S}_{2}$ line intensities show a large scatter that can mask any differences in line intensities between different samples of objects. Therefore it is preferable to compare not the line intensities but the ratio of the line intensities.

Panel a of Fig. 6 shows the $\mathrm{N}_{2} / \mathrm{R}_{2}$ line ratio as a function of $\mathrm{N}_{2}$ line intensity for our samples of slit spectra of $\mathrm{H}$ II regions in nearby galaxies, for the SDSS fibre spectra (catalogues SDSS-A and SDSS-B), and for the MaNGA spaxel spectra. The symbols represent the mean values in bins of $0.05 \mathrm{dex}$ in $\mathrm{N}_{2}$. The increase of $\mathrm{N}_{2}$ or equal increases of $\mathrm{N}_{2}$ and $\mathrm{R}_{2}$ would result in a shift of the points along the $\mathrm{X}$-axis towards a larger $\mathrm{N}_{2}$ value. The increase of $R_{2}$ or different increases of $R_{2}$ and $N_{2}$ would result in a shift of the points along the Y-axis. Inspection of Fig. 6 shows that the $\mathrm{N}_{2} / \mathrm{R}_{2}-\mathrm{N}_{2}$ diagrams for the SDSS and the MaNGA objects are in agreement with each other but there is some difference to those for the $\mathrm{H}$ II regions in nearby galaxies in the sense that there is shift of the $\mathrm{N}_{2} / \mathrm{R}_{2}-\mathrm{N}_{2}$ diagrams for the SDSS and the MaNGA objects relative to the $\mathrm{N}_{2} / \mathrm{R}_{2}-\mathrm{N}_{2}$ diagram for the slit spectra of $\sim 0.1$ dex along the $X$ - or $Y$-axis.

It has been known for a long time that there is no one-to-one correspondence between the oxygen and nitrogen abundances in galaxies since there is a time delay in the enrichment of the interstellar medium in nitrogen as compared to oxygen. The nitrogen-to-oxygen ratio at a given oxygen abundance depends on the star formation history in a galaxy, that is, N/O is an indicator of the time that has elapsed since the last episode of star formation (Edmunds \& Pagel 1978). Thus, the difference in the $\mathrm{N}_{2} / \mathrm{R}_{2}-\mathrm{N}_{2}$ diagrams may seem to suggest that there are differences in the star formation histories of the sample of nearby galaxies and in the galaxy samples between the SDSS and MaNGA surveys. On the other hand, the difference in the $\mathrm{N}_{2} / \mathrm{R}_{2}-$ $\mathrm{N}_{2}$ diagrams may suggest that the strengths of the $\mathrm{N}_{2}$ and/or $\mathrm{R}_{2}$ in the spaxel (fibre) spectra are increased by the contribution of the radiation of the DIG. If this is the case then the increase of the strengths of the $\mathrm{N}_{2}$ and/or $\mathrm{R}_{2}$ does not exceed a factor of $\sim 1.3$.

Panels $b$ and $c$ of Fig. 6 show the $S_{2} / R_{2}$ and $N_{2} / S_{2}$ line ratios as a function of $\mathrm{N}_{2}$ for those samples of objects. Inspection of these two panels shows that the $S_{2} / R_{2}-N_{2}$ and $N_{2} / S_{2}-N_{2}$ diagrams for the MaNGA galaxies differ from the ones for the SDSS galaxies and for the nearby galaxies. The difference is maximized between the $\mathrm{S}_{2} / \mathrm{R}_{2}-\mathrm{N}_{2}$ (and $\mathrm{N}_{2} / \mathrm{S}_{2}-\mathrm{N}_{2}$ ) diagrams for the MaNGA and the nearby galaxies. The difference changes with $\mathrm{N}_{2}$. The difference in $\mathrm{S}_{2} / \mathrm{R}_{2}$ and $\mathrm{N}_{2} / \mathrm{S}_{2}$ is around $0.3 \mathrm{dex}$ at $\log \mathrm{N}_{2} \sim-0.5$. If the contribution of the radiation of the diffuse ionized gas to the spaxel (fibre) spectra is responsible for the differences in the $S_{2} / R_{2}$ and $N_{2} / S_{2}$ line ratios then this shows that the enhancement of the strength of $S_{2}$ due to the contribution of the DIG amounts to up to a factor of two.
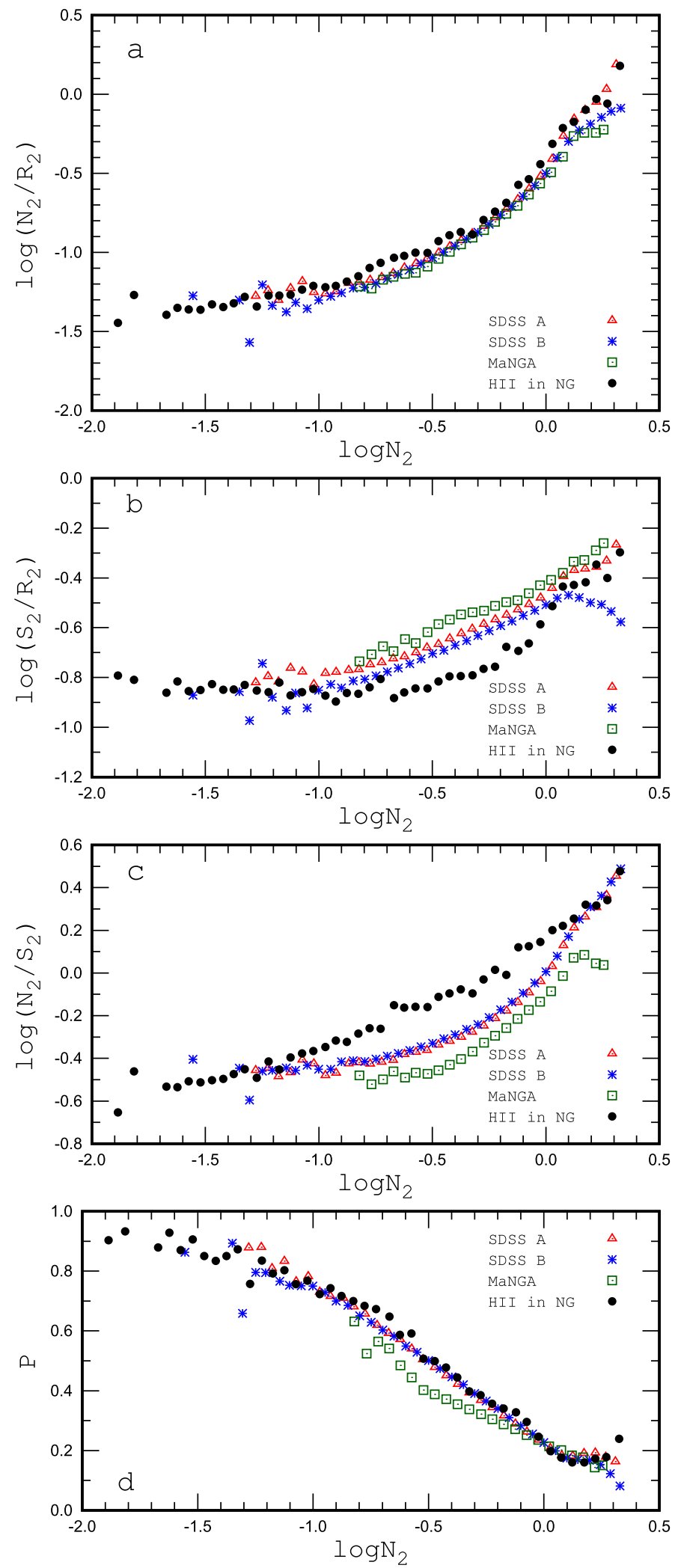

Fig. 6. Panel a: nitrogen to oxygen line ratio $\mathrm{N}_{2} / \mathrm{R}_{2}$ as a function of the $\mathrm{N}_{2}$ line intensity for the SDSS fibre spectra (catalogues SDSS-A and SDSS-B), for the spaxel MaNGA spectra, and for the slit spectra of $\mathrm{H}$ II regions in nearby galaxies. The symbols represent the mean values for objects in bins of $0.05 \mathrm{dex}$ in $\log \mathrm{N}_{2}$. Panels $b, c$, and $d$ : same but for the sulphur to oxygen line ratio $S_{2} / R_{2}$, for the nitrogen to sulphur line ratio $\mathrm{N}_{2} / \mathrm{S}_{2}$, and for the excitation parameter $P$, respectively. 

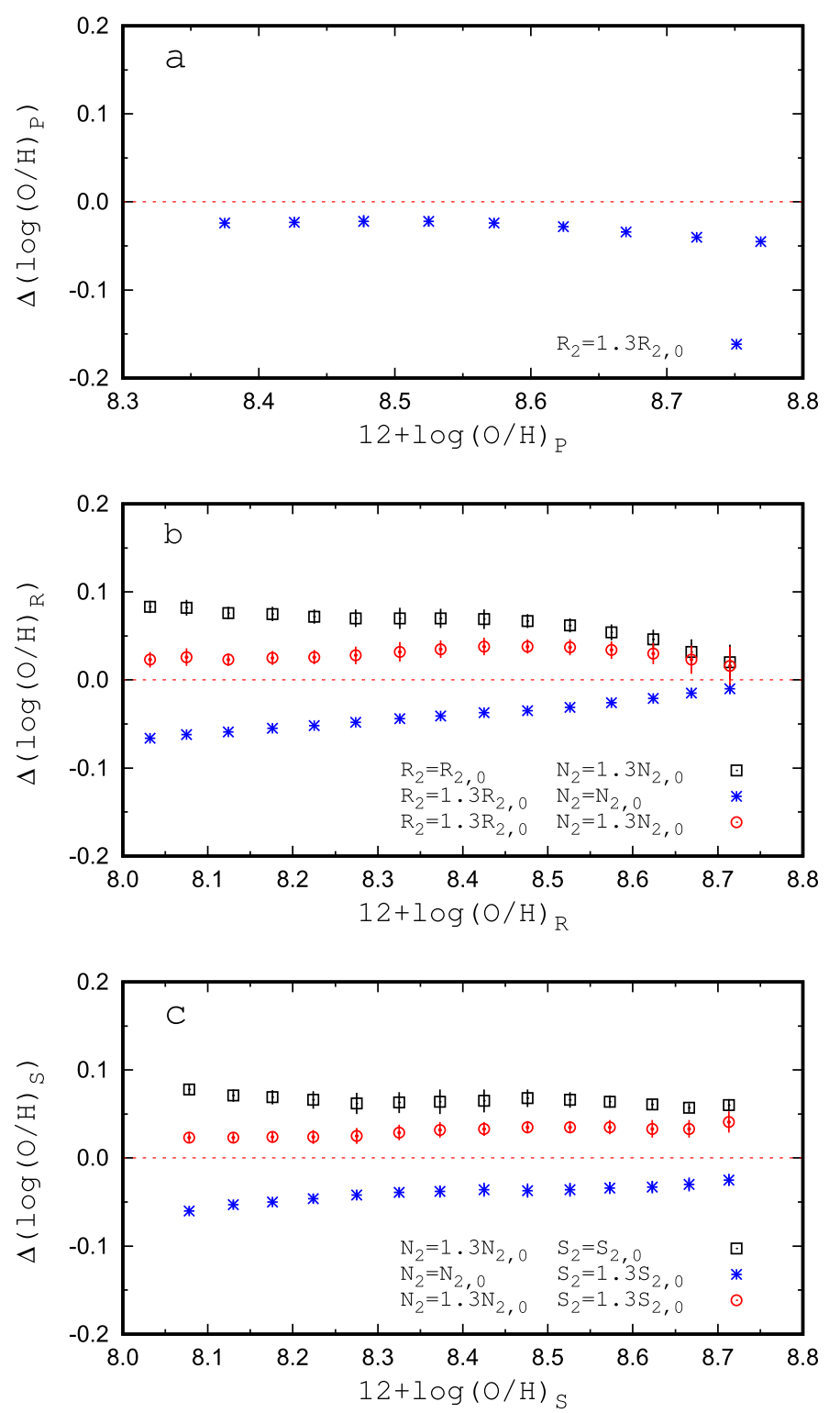

Fig. 7. Influence of the increase of the emission-line fluxes on the oxygen abundances obtained through different calibrations. Panel a: difference between oxygen abundances in $\mathrm{H}$ II regions in nearby galaxies obtained with the original $R_{2}=R_{2,0}$ emission line and with the emission line $R_{2}=1.3 R_{2,0}$ increased by a factor of 1.3 . The symbols represent the mean values of the abundance differences for objects in bins of $0.05 \mathrm{dex}$ in $(\mathrm{O} / \mathrm{H})$. The bars (comparable with the symbol size) denote the mean values of the scatter of the differences in the bins. Panel $b$ : influence of the increase of the $\mathrm{R}_{2}$ and $\mathrm{N}_{2}$ emission-line fluxes on the oxygen abundances $(\mathrm{O} / \mathrm{H})_{R}$. Panel $c$ : influence of the increase of the $\mathrm{N}_{2}$ and $\mathrm{S}_{2}$ emission-line fluxes on the oxygen abundances $(\mathrm{O} / \mathrm{H})_{S}$.

Panel $\mathrm{d}$ of Fig. 6 shows the excitation parameter $P$ as a function of $\mathrm{N}_{2}$ for those samples of objects. Again, the largest difference between the excitation parameters at a given $\mathrm{N}_{2}$ occurs between the MaNGA spaxel spectra and H II regions in nearby galaxies.

\subsubsection{Influence of the line strength increase on the obtained abundances}

Before comparing the abundances determined through different calibrations from the $\mathrm{HII}$ region slit spectra, the SDSS fibre
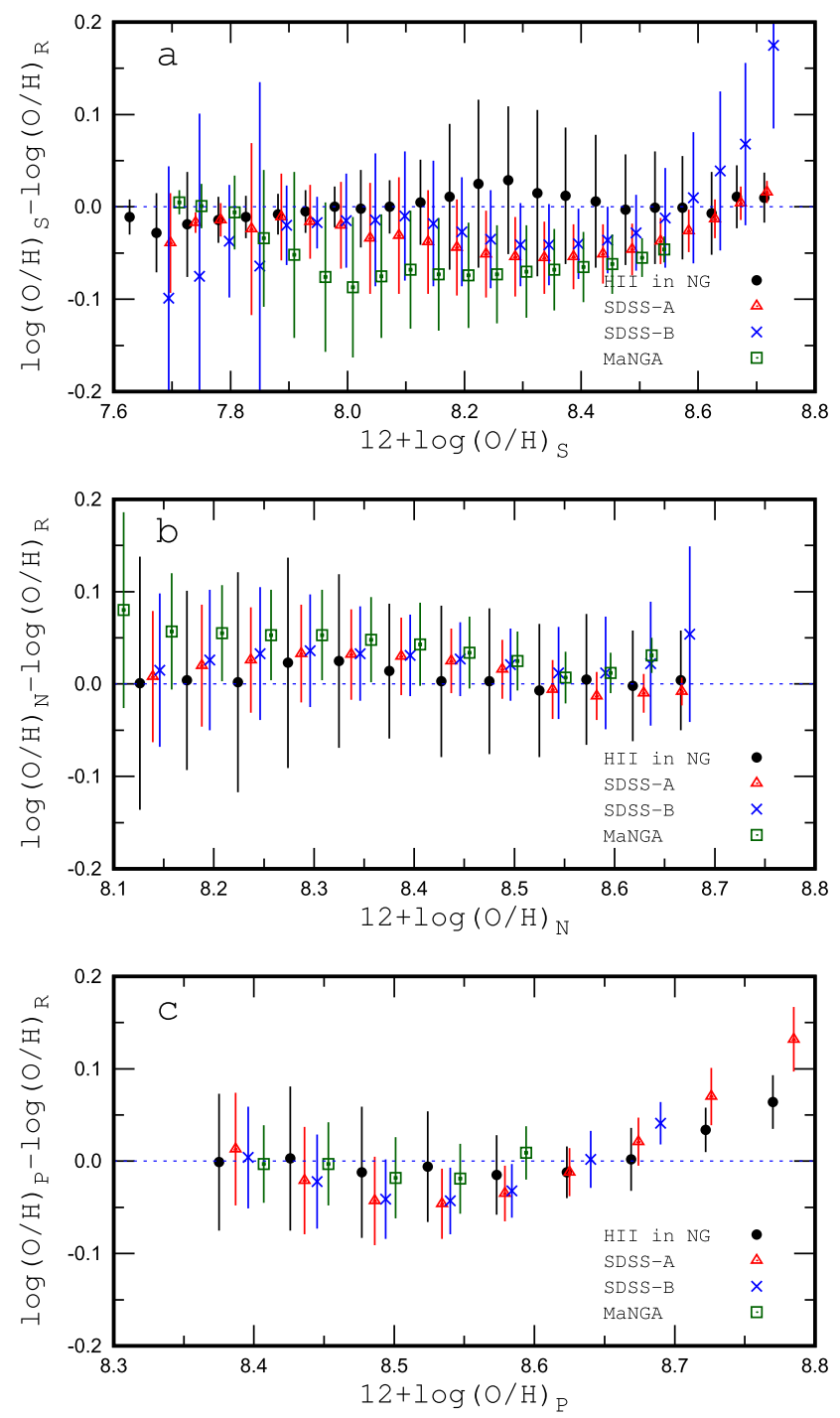

Fig. 8. Comparison between oxygen abundances determined through different calibrations based on the slit spectra of H II regions in nearby galaxies, the fibre SDSS spectra (with lines from the SDSS-A and SDSS-B catalogues), and the MaNGA spaxel spectra. The symbols represent the mean values of the differences for objects in bins of 0.05 dex in $\mathrm{O} / \mathrm{H}$, and the bars show the scatter of differences in these bins.

spectra, and the MaNGA spaxel spectra, we examine the influence of the increase of the emission-line strengths on the oxygen abundances obtained through different calibrations. This can be examined by comparing oxygen abundances in H II regions in nearby galaxies obtained with the original strengths of the emission lines and with the increased strengths. The artificial increase of the emission-line intensities can be considered as a rough simulation of the contribution of the radiation of the diffuse ionized gas to the composite spectra.

It is evident that the change of the value of the $(\mathrm{O} / \mathrm{H})_{N}$ abundance depends on the change of $\mathrm{N}_{2}$, but does not depend on the change of $R_{2}$ and $S_{2}$. It can easily be seen from Eq. (6) that the increase of the strength of the $\mathrm{N}_{2}$ by a factor of 1.3 results in an increase of $(\mathrm{O} / \mathrm{H})_{N}$ by 0.06 dex. It turn, the change of the value of the $(\mathrm{O} / \mathrm{H})_{P}$ abundance depends on the change of $\mathrm{R}_{2}$, but does not depend on the change of $\mathrm{N}_{2}$ and $\mathrm{S}_{2}$. Panel a of Fig. 7 shows the difference between the oxygen abundances $(\mathrm{O} / \mathrm{H})_{P}$ in $\mathrm{H}$ II regions in nearby galaxies obtained with the original emission line $R_{2}=R_{2,0}$ and with the emission line $R_{2}=1.3 R_{2,0}$ 

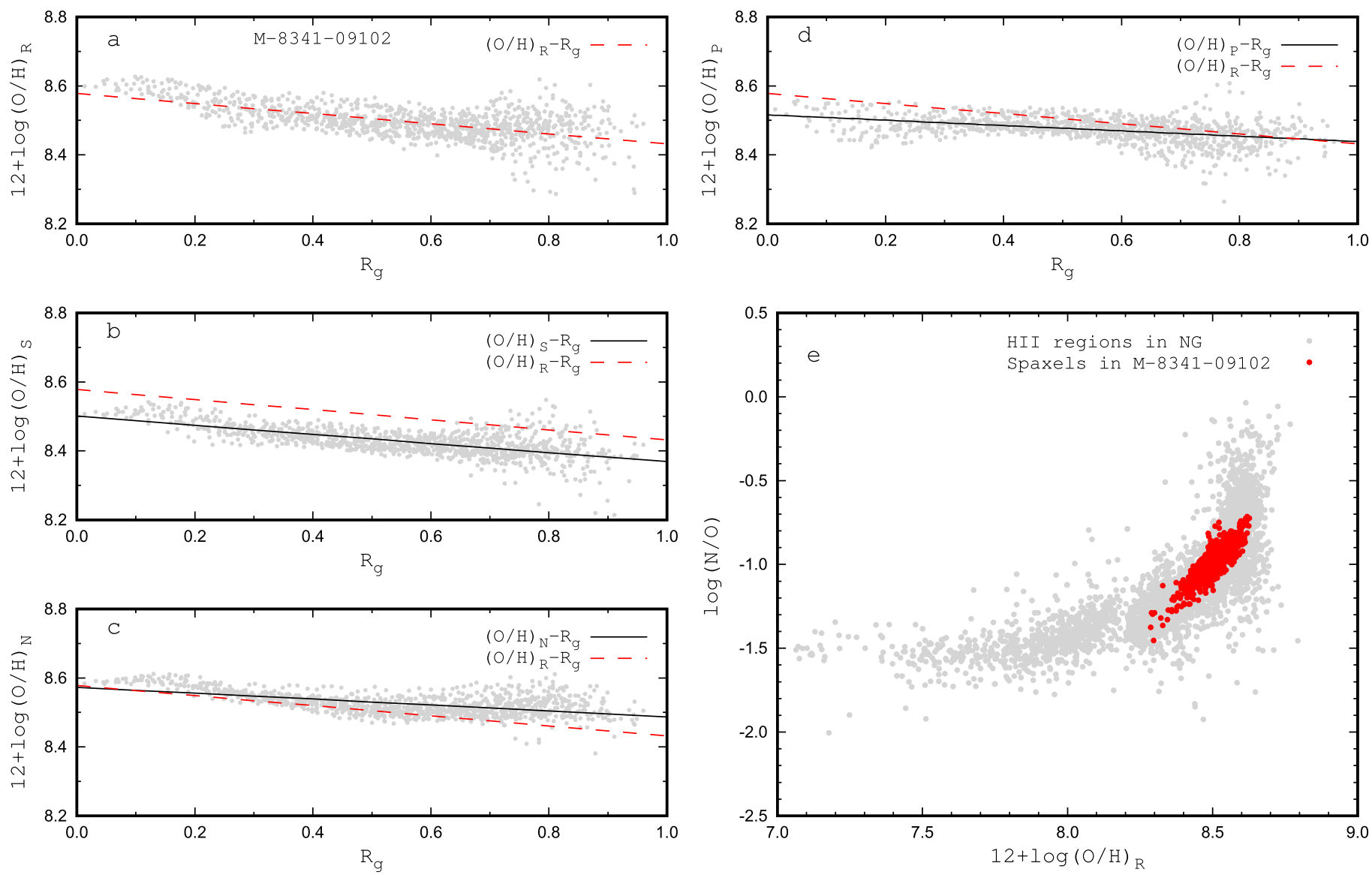

Fig. 9. Panels $a, b, c$, and $d$ : radial distributions of the oxygen abundances determined through the different calibrations in the disc of the MaNGA galaxy M-8341-09102. The grey points are abundances in the individual spaxels. The line is the best fit to those data. The dashed line in each panel is the $R_{g}-(\mathrm{O} / \mathrm{H})_{R}$ relation. Panel $e:(\mathrm{O} / \mathrm{H})_{R}-\mathrm{N} / \mathrm{O}$ diagram. The grey points are abundances in the $\mathrm{H}$ II regions in the nearby galaxies; the red points are abundances in the individual spaxels of the MaNGA galaxy M-8341-09102.

increased by a factor of 1.3 in each individual spectrum. The symbols represent the mean values of the abundance differences in bins of 0.05 dex in $(\mathrm{O} / \mathrm{H})_{P}$. The bars (comparable with the symbol size) denote the mean values of the scatter of the differences in the bins. Panel a of Fig. 7 shows that the increase of the $\mathrm{R}_{2}$ emission-line intensity by a factor of 1.3 results in a decrease of the $(\mathrm{O} / \mathrm{H})_{P}$ abundance by around 0.03 dex.

Panel b of Fig. 7 shows the influence of the increase of the $\mathrm{R}_{2}$ and $\mathrm{N}_{2}$ emission-line strengths on the $(\mathrm{O} / \mathrm{H})_{R}$ abundance. One can see that an increase of the $\mathrm{R}_{2}$ emission-line intensity by a factor of 1.3 leads to a decrease of the $(\mathrm{O} / \mathrm{H})_{R}$ abundance by around $0.06 \operatorname{dex}$ at $12+\log (\mathrm{O} / \mathrm{H}) \sim 8.1$ and by around 0.015 dex at $12+\log (\mathrm{O} / \mathrm{H}) \sim 8.65$. Increasing the $\mathrm{N}_{2}$ emissionline intensity by a factor of 1.3 results in an increase of the $(\mathrm{O} / \mathrm{H})_{R}$ abundance by around 0.07 dex at $12+\log (\mathrm{O} / \mathrm{H}) \sim 8.1$ and by around 0.04 dex at $12+\log (\mathrm{O} / \mathrm{H}) \sim 8.65$. An increase of the $\mathrm{R}_{2}$ line intensity results in the opposite effect on the $(\mathrm{O} / \mathrm{H})_{R}$ abundance as compared to the increase of the $\mathrm{N}_{2}$ line intensity. Thus, an increase of both the $\mathrm{R}_{2}$ and $\mathrm{N}_{2}$ line intensities by a factor of 1.3 results in an increase of the $(\mathrm{O} / \mathrm{H})_{R}$ abundance of $\sim 0.04$ dex

Panel c of Fig. 7 shows the influence of the increase of the $\mathrm{N}_{2}$ and $\mathrm{S}_{2}$ emission-line strengths on the $(\mathrm{O} / \mathrm{H})_{S}$ abundance. Again, an increase of the $S_{2}$ line intensity results in the opposite effect on the $(\mathrm{O} / \mathrm{H})_{S}$ abundance as compared to the increase of the $\mathrm{N}_{2}$ line intensity, and the increase of both the $\mathrm{S}_{2}$ and $\mathrm{N}_{2}$ line intensities by a factor of 1.3 leads to an increase of the $(\mathrm{O} / \mathrm{H})_{S}$ abundance by $\sim 0.04$ dex.
Thus, an increase of the $\mathrm{R}_{2}, \mathrm{~N}_{2}$, and $\mathrm{S}_{2}$ line strengths by a factor of 1.3 results in an increase of the $(\mathrm{O} / \mathrm{H})_{N}$ abundance by as much as $0.06 \mathrm{dex}$, a decrease of the $(\mathrm{O} / \mathrm{H})_{P}$ abundance of around $0.03 \mathrm{dex}$, and an increase of the $(\mathrm{O} / \mathrm{H})_{R}$ and $(\mathrm{O} / \mathrm{H})_{S}$ abundances by $\sim 0.04$ dex.

\subsubsection{Comparison between abundances determined through different calibrations in our samples of objects}

Panel a of Fig. 8 shows the differences between the $(\mathrm{O} / \mathrm{H})_{S}$ and $(\mathrm{O} / \mathrm{H})_{R}$ oxygen abundances determined from the slit spectra of $\mathrm{H}$ II regions in nearby galaxies, from the SDSS fibre spectra (for the SDSS-A and SDSS-B catalogues), and from the MaNGA spaxel spectra. The symbols represent the mean values of the differences for objects in bins of 0.05 dex in $(\mathrm{O} / \mathrm{H})_{S}$. The bars denote the mean values of the scatter of the differences for objects in the bins. Examination of panel a of Fig. 8 shows that the difference between the $(\mathrm{O} / \mathrm{H})_{S}$ and $(\mathrm{O} / \mathrm{H})_{R}$ oxygen abundances determined from the slit spectra of $\mathrm{H}$ II regions in nearby galaxies is rather small, usually lower than 0.02 dex.

The $(\mathrm{O} / \mathrm{H})_{R}-(\mathrm{O} / \mathrm{H})_{S}$ diagrams for the SDSS objects obtained with the lines from the SDSS-A and SDSS-B catalogues are similar for metallicities from $12+\log (\mathrm{O} / \mathrm{H})_{S} \sim 7.9$ to $12+$ $\log (\mathrm{O} / \mathrm{H})_{S} \sim 8.6$ and they differ significantly at lower $(12+$ $\left.\log (\mathrm{O} / \mathrm{H})_{S} \lesssim 7.9\right)$ and higher $\left(12+\log (\mathrm{O} / \mathrm{H})_{S} \gtrsim 8.6\right)$ metallicities. Thus, the comparison between $(\mathrm{O} / \mathrm{H})_{R}$ and $(\mathrm{O} / \mathrm{H})_{S}$ abundances in the SDSS objects is justified for objects with metallicities from $12+\log (\mathrm{O} / \mathrm{H})_{S} \sim 7.9$ to $12+\log (\mathrm{O} / \mathrm{H})_{S} \sim 8.6$ 
only. The $(\mathrm{O} / \mathrm{H})_{S}$ abundances are lower than the $(\mathrm{O} / \mathrm{H})_{R}$ abundances, and the difference is as large as $\sim 0.05$ dex for objects with metallicities from $12+\log (\mathrm{O} / \mathrm{H}) \sim 8.2$ to $12+\log (\mathrm{O} / \mathrm{H})$ $\sim$ 8.5. The $(\mathrm{O} / \mathrm{H})_{S}$ abundances in the MaNGA objects are also lower than the $(\mathrm{O} / \mathrm{H})_{R}$ abundances, and the difference is larger (up to around $0.1 \mathrm{dex}$ ) than for the SDSS objects. This is not surprising since the $S_{2}$ line intensity is significantly enhanced in the SDSS fibre and MaNGA spaxel spectra.

Panel b of Fig. 8 shows the differences between the $(\mathrm{O} / \mathrm{H})_{N}$ and $(\mathrm{O} / \mathrm{H})_{R}$ abundances for our samples of objects. The applicability of our $N$ calibration is restricted by the condition $\log \mathrm{N}_{2} \gtrsim$ -0.6 , hence the $(\mathrm{O} / \mathrm{H})_{N}$ abundances in low-metallicity objects cannot be determined. One can see that the difference between the $(\mathrm{O} / \mathrm{H})_{N}$ and $(\mathrm{O} / \mathrm{H})_{R}$ abundances for the $\mathrm{H}$ II regions in nearby galaxies is small, usually lower than 0.01 dex, except for objects with abundances of $12+\log (\mathrm{O} / \mathrm{H}) \sim 8.3$ where the difference is around 0.025 dex. Again, the $(\mathrm{O} / \mathrm{H})_{R}-(\mathrm{O} / \mathrm{H})_{N}$ diagrams for the SDSS objects obtained with lines from the SDSS-A and SDSS-B catalogues are similar to each other for metallicities $12+\log (\mathrm{O} / \mathrm{H})_{N} \lesssim 8.6$, but differ for higher metallicities. The $(\mathrm{O} / \mathrm{H})_{N}$ abundances are higher than the $(\mathrm{O} / \mathrm{H})_{R}$ abundances, and the difference is within $0.03 \mathrm{dex}$, which is comparable to the maximum difference between the $(\mathrm{O} / \mathrm{H})_{N}$ and $(\mathrm{O} / \mathrm{H})_{R}$ abundances for the $\mathrm{H}$ II regions in nearby galaxies. The $(\mathrm{O} / \mathrm{H})_{N}$ abundances in the MaNGA objects are also higher than the $(\mathrm{O} / \mathrm{H})_{R}$ abundances. The difference increases with decreasing metallicity and is around 0.05 dex for objects with $12+\log (\mathrm{O} / \mathrm{H})_{N} \lesssim 8.3$.

Close examination of Figs. 8 and 6 suggests that the discrepancy between the $(\mathrm{O} / \mathrm{H})_{N}$ and $(\mathrm{O} / \mathrm{H})_{R}$ abundances for the MaNGA galaxies can be explained by the intrinsic properties of the $\mathrm{N}_{2}$ calibration. Indeed, the $N$ calibration can produce an incorrect $(\mathrm{O} / \mathrm{H})_{N}$ even if the strength of $\mathrm{N}_{2}$ is correct. Like any other $1 \mathrm{D}$ calibration, the $\mathrm{N}_{2}$ calibration encounters the following problem. The $\mathrm{N}_{2}$ line intensity in the spectrum of $\mathrm{H}$ II region depends not only on the oxygen abundance $(\mathrm{O} / \mathrm{H})$ but also on its excitation $P$ and the nitrogen-to-oxygen abundance ratio $\mathrm{N} / \mathrm{O}$, that is, $\mathrm{N}_{2}=f(\mathrm{O} / \mathrm{H}, P, \mathrm{~N} / \mathrm{O})$ or $(\mathrm{O} / \mathrm{H})_{N}=f\left(\mathrm{~N}_{2}, P, \mathrm{~N} / \mathrm{O}\right)$. The calibrating data points occupy a wide band in the $\mathrm{O} / \mathrm{H}-\mathrm{N}_{2}$ diagram, that is, the scatter around the $1 \mathrm{D}$ calibration relation $\mathrm{O} / \mathrm{H}=f\left(\mathrm{~N}_{2}\right)$ is large (Pettini \& Pagel 2004; Marino et al. 2013). The deviation of the calibrating H II region from the $\mathrm{O} / \mathrm{H}=f\left(\mathrm{~N}_{2}\right)$ relation depends on its excitation and the $\mathrm{N} / \mathrm{O}$ abundance ratio. Of course, the uncertainties in the oxygen abundances in the calibrating data points also contribute to the scatter around the $\mathrm{O} / \mathrm{H}=f\left(\mathrm{~N}_{2}\right)$ relation. The calibration relation $(\mathrm{O} / \mathrm{H})_{N}$ $=f\left(\mathrm{~N}_{2}\right)$ produces the correct $(\mathrm{O} / \mathrm{H})_{N}$ abundance in a target region (spaxel) if only its excitation $P$ and nitrogen-to-oxygen abundance ratio N/O correspond to those parameters in the calibrating points (with a given value of $\mathrm{N}_{2}$ ) located close to the $(\mathrm{O} / \mathrm{H})=f\left(\mathrm{~N}_{2}\right)$ relation in the $\mathrm{O} / \mathrm{H}-\mathrm{N}_{2}$ diagram. If the excitation $P$ and/or the nitrogen-to-oxygen abundance ratio N/O in the target spaxel region differs from those values in the calibrating points located close to the $\mathrm{O} / \mathrm{H}=f\left(\mathrm{~N}_{2}\right)$ relation then the $\mathrm{N}_{2}$ calibration produces incorrect $(\mathrm{O} / \mathrm{H})_{N}$ abundance. Then the disagreement between the $(\mathrm{O} / \mathrm{H})_{N}$ and $(\mathrm{O} / \mathrm{H})_{R}$ abundances for the MaNGA galaxies (panel b of Fig. 8) can be explained by incorrect $(\mathrm{O} / \mathrm{H})_{N}$ abundances caused by the systematic difference in the excitations $P$ of the MaNGA spaxel spectra and H II regions in nearby galaxies (panel d of Fig. 6) used as the calibrating data points in deriving the $\mathrm{O} / \mathrm{H}-\mathrm{N}_{2}$ relation. This expectation will be checked below through the comparison between the radial distributions of the $(\mathrm{O} / \mathrm{H})_{N}$ and $(\mathrm{O} / \mathrm{H})_{R}$ abundances in the MaNGA galaxies.
Panel c of Fig. 8 shows the differences between the $(\mathrm{O} / \mathrm{H})_{P}$ and $(\mathrm{O} / \mathrm{H})_{R}$ abundances for our samples of objects. The applicability of the upper-branch $P$ calibration is restricted by the condition $12+\log (\mathrm{O} / \mathrm{H}) \gtrsim 8.35$. Therefore the $(\mathrm{O} / \mathrm{H})_{P}$ abundances are determined for high-metallicity objects only. On the other hand, the $(\mathrm{O} / \mathrm{H})_{P}$ abundances at high metallicities $(12+\log (\mathrm{O} / \mathrm{H}) \gtrsim 8.55)$ can involve a large error due to the uncertainty in the data reduction and emission line measurements; see Fig. 5. Thus, the $(\mathrm{O} / \mathrm{H})_{P}$ abundances are more or less reliable in a narrow interval of metallicities only. Therefore, a reliable distribution of the $(\mathrm{O} / \mathrm{H})_{P}$ abundances can be found only in a small number of galaxies.

It should be noted that the difference between abundances estimated through different calibrations for individual objects can be significantly larger than the mean value of the differences. The mean scatter of the differences is shown by the error bars in Fig. 8.

\section{Abundance distributions in the MaNGA galaxies}

The $(\mathrm{O} / \mathrm{H})_{R},(\mathrm{O} / \mathrm{H})_{S},(\mathrm{O} / \mathrm{H})_{N}$, and $(\mathrm{O} / \mathrm{H})_{R}$ abundances were estimated from the spaxel spectra in the discs of the MaNGA galaxies of our sample. The radial abundance distributions were fitted by linear relations. The obtained $(\mathrm{O} / \mathrm{H})-R_{g}$ relation produces the central (intersect) abundance and abundance at the optical radius $R_{25}$ (also the intersect value). Since the $P$ calibration is applicable to high-metallicity objects only, the $(\mathrm{O} / \mathrm{H})_{P}-R_{g}$ relation was determined for spaxels with galactocentric distances of less than $R^{*}$, where $R^{*}$ is the radius at which the $12+\log (\mathrm{O} / \mathrm{H})_{R}=8.4$. If $R^{*}<0.3 R_{25}$ then the $(\mathrm{O} / \mathrm{H})_{P}-R_{g}$ relation was not determined and the central abundance could not be estimated. If $0.3 R_{25}<R^{*}<0.7 R_{25}$ then the $(\mathrm{O} / \mathrm{H})_{P}-R_{g}$ relation was used to estimate the central abundance but the abundance at the optical radius was not measured. If $R^{*}>0.7 R_{25}$ then both the central abundance and the abundance at the optical radius were estimated from the $(\mathrm{O} / \mathrm{H})_{P}-R_{g}$ relation.

The $(\mathrm{O} / \mathrm{H})_{R}-\mathrm{N} / \mathrm{O}$ diagram was also constructed for each MaNGA galaxy and compared to the diagram for the $\mathrm{H}$ II regions in nearby galaxies. The N/O abundances ratio was estimated using the N/O calibration relation from Pilyugin \& Grebel (2016). This diagram serves for visual control of the validity of the obtained abundances.

Panel a of Fig. 9 shows the radial distribution of the $(\mathrm{O} / \mathrm{H})_{R}$ abundances across the disc of the MaNGA galaxy M-834109102. The grey points are abundances in the individual spaxels. The dashed line is the best fit to those data. Panels b, c, and d of Fig. 9 show the radial distributions of the $(\mathrm{O} / \mathrm{H})_{S},(\mathrm{O} / \mathrm{H})_{N}$, and $(\mathrm{O} / \mathrm{H})_{P}$ abundances in the disc of M-8341-09102, respectively. The grey points are abundances in the individual spaxels, and the solid line depicts the best fit to those data. The dashed line in panels b, c, and d is the $R_{g}-(\mathrm{O} / \mathrm{H})_{R}$ relation from the panel a. Panel e shows the $(\mathrm{O} / \mathrm{H})_{R}-\mathrm{N} / \mathrm{O}$ diagram. The grey points denote abundances in the HII regions in the nearby galaxies; the red points are abundances in the individual spaxels of the MaNGA galaxy M-8341-09102.

Panel a of Fig. 10 shows the comparison between the $(\mathrm{O} / \mathrm{H})_{s}$ and $(\mathrm{O} / \mathrm{H})_{R}$ abundances for our sample of MaNGA galaxies. The circles mark the central (intersect) oxygen abundances. The plus signs mark the abundances (intersect values) at the optical radius $R_{25}$. The short-dashed line is that of equal values. For comparison, the solid line shows the $(\mathrm{O} / \mathrm{H})_{s}$ versus $(\mathrm{O} / \mathrm{H})_{R}$ abundances (mean values in bins of $0.05 \mathrm{dex}$ in $(\mathrm{O} / \mathrm{H})_{R}$ ) for our sample of $\mathrm{H}$ II regions in the nearby galaxies). The long-dashed line shows the 

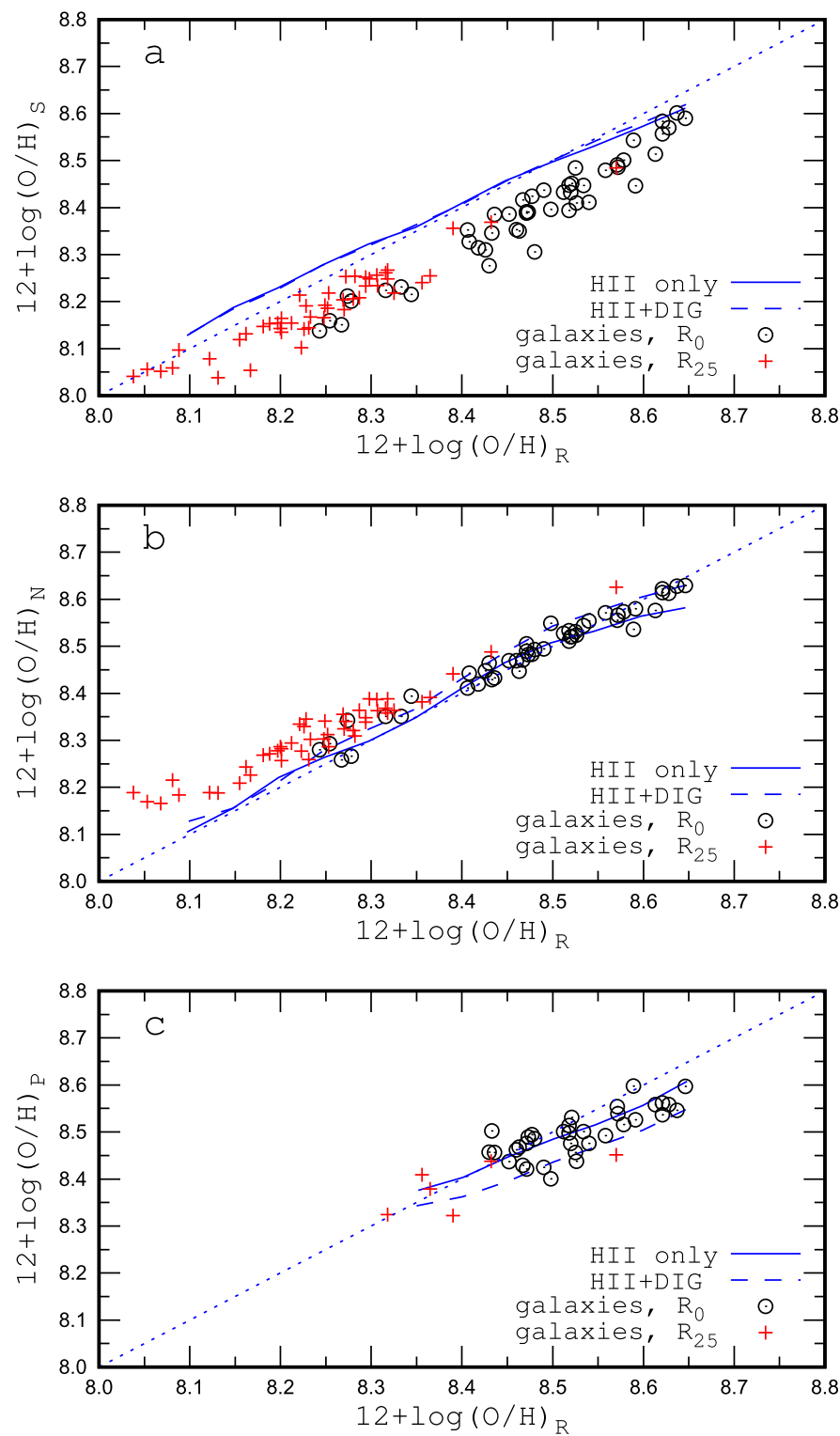

Fig. 10. Comparison of the $(\mathrm{O} / \mathrm{H})_{R}$ oxygen abundances with abundances estimated through other calibrations for our sample of MaNGA galaxies. The circles in each panel show the central (intersect) oxygen abundances. The plus symbols indicate abundances (intersect values) at the optical radius $R_{25}$. The solid line shows the oxygen abundances for the sample of $\mathrm{H}$ II regions in the nearby galaxies. The long-dashed line shows the oxygen abundances for those $\mathrm{H}$ II regions obtained with the lines $R_{2}, N_{2}$, and $S_{2}$ increased by a factor of 1.3 to simulate the DIG contribution to the spectra. The short-dashed line indicates unity.

$(\mathrm{O} / \mathrm{H})_{s}$ versus $(\mathrm{O} / \mathrm{H})_{R}$ abundances for those $\mathrm{H}$ II regions obtained with the lines $R_{2}, N_{2}$, and $S_{2}$ increased by a factor of 1.3 to simulate the DIG contribution to the spectra.

Panel a of Fig. 10 shows that the $(\mathrm{O} / \mathrm{H})_{S}$ abundances are lower than the $(\mathrm{O} / \mathrm{H})_{R}$ abundances both in the centres and at the optical radii of the MaNGA galaxies. The increase of the lines $\mathrm{N}_{2}$ and $\mathrm{S}_{2}$ by a factor of 1.3 cannot explain the difference between the $(\mathrm{O} / \mathrm{H})_{S}$ and $(\mathrm{O} / \mathrm{H})_{R}$ abundances. This is not surprising. Indeed, panels $b$ and $c$ of Fig. 6 show that the sulphur line $S_{2}$ is increased in the MaNGA spectra by a factor up to $\sim 2$.

Panel b of Fig. 10 shows the comparison between the $(\mathrm{O} / \mathrm{H})_{N}$ and $(\mathrm{O} / \mathrm{H})_{R}$ abundances for our sample of MaNGA galaxies. The circles stand for the central abundances; the plus signs are the abundances at the optical radii $R_{25}$. Again, the solid line shows $(\mathrm{O} / \mathrm{H})_{N}$ as a function of the $(\mathrm{O} / \mathrm{H})_{R}$ abundance for our sample of $\mathrm{H}$ II regions in nearby galaxies, and the long-dashed line denotes the oxygen abundances for $\mathrm{H}$ II regions obtained with the lines $\mathrm{R}_{2}$ and $\mathrm{N}_{2}$ increased by a factor of 1.3 to simulate the DIG contribution to the spectra.

Panel b of Fig. 10 shows that the central $(\mathrm{O} / \mathrm{H})_{N}$ and $(\mathrm{O} / \mathrm{H})_{R}$ abundances in the MaNGA galaxies are close to each other. They are compatible with the $(\mathrm{O} / \mathrm{H})_{N}-(\mathrm{O} / \mathrm{H})_{R}$ diagram for $\mathrm{H}$ II regions in nearby galaxies. The increase of the lines $R_{2}$ and/or $\mathrm{N}_{2}$ in the MaNGA spaxel spectra is less than a factor of 1.3 (if any). This is strong evidence showing that the central $(\mathrm{O} / \mathrm{H})_{N}$ and $(\mathrm{O} / \mathrm{H})_{R}$ abundances are relatively reliable.

At the same time, panel $b$ of Fig. 10 shows that the $(\mathrm{O} / \mathrm{H})_{N}$ abundances at the optical radii are systematically higher than the $(\mathrm{O} / \mathrm{H})_{R}$ abundances. We suggest above that the discrepancy between the $(\mathrm{O} / \mathrm{H})_{N}$ and $(\mathrm{O} / \mathrm{H})_{R}$ abundances determined from the MaNGA spectra can be explained by the fact that the $N$-calibration produces incorrect abundances if the excitation $P$ and/or the nitrogen-to-oxygen abundance ratio, N/O, in an object differs from the values in the calibrating points located in the $(\mathrm{O} / \mathrm{H})_{R}-\mathrm{N}_{2}$ diagram close to the $(\mathrm{O} / \mathrm{H})=f\left(\mathrm{~N}_{2}\right)$ relation. We examine this expectation here.

Panel a of Fig. 11 shows the excitation parameter $P$ as a function of the $\mathrm{N}_{2}$ line intensity for the spaxels in the central regions $\left(R_{g}<0.1\right)$ of several MaNGA galaxies. The mean values of $P$ in bins of 0.1 dex in $\mathrm{N}_{2}$ are presented. The solid line shows the excitation parameter $P$ as a function of $N_{2}$ for our $\mathrm{H}$ II regions in nearby galaxies with $0.03>\log (\mathrm{O} / \mathrm{H})_{N}-\log (\mathrm{O} / \mathrm{H})_{R}>-0.03$. The HII regions with correct $(\mathrm{O} / \mathrm{H})_{N}$ abundances are located close to the $\mathrm{O} / \mathrm{H}=f\left(\mathrm{~N}_{2}\right)$ relation in the $(\mathrm{O} / \mathrm{H})_{R}-\mathrm{N}_{2}$ diagram. The long-dashed line shows the excitation parameter $P$ as a function of the $\mathrm{N}_{2}$ for $\mathrm{H}$ II regions with $0.09>\log (\mathrm{O} / \mathrm{H})_{N}-\log (\mathrm{O} / \mathrm{H})_{R}>$ 0.03 , and the short-dashed line for H II regions with $-0.03>$ $\log (\mathrm{O} / \mathrm{H})_{N}-\log (\mathrm{O} / \mathrm{H})_{R}>-0.09$. Those $\mathrm{H}$ II regions with incorrect $(\mathrm{O} / \mathrm{H})_{N}$ abundances are shifted from the $\mathrm{O} / \mathrm{H}=f\left(\mathrm{~N}_{2}\right)$ relation in the $(\mathrm{O} / \mathrm{H})_{R}-\mathrm{N}_{2}$ diagram. Panel c shows the same as Panel a but for spaxels with galactocentric distances from 0.775 to $0.825 R_{g}$.

Panel b of Fig. 11 shows the $\mathrm{N}_{2} / \mathrm{R}_{2}$ line ratio as a function of the $\mathrm{N}_{2}$ line intensity for spaxels in central regions $\left(R_{g}<0.1\right)$ of several MaNGA galaxies. Panel d shows the same as Panel b but for spaxels with galactocentric distances from 0.775 to $0.825 R_{g}$.

Examination of Fig. 11 shows that the excitation parameter $P$ and the $\mathrm{N}_{2} / \mathrm{R}_{2}$ line ratio for the spaxels in central regions of the MaNGA galaxies are close to the values in the $\mathrm{H}$ II regions in nearby galaxies with similar $(\mathrm{O} / \mathrm{H})_{N}$ and $(\mathrm{O} / \mathrm{H})_{R}$ abundances. Therefore, the central $(\mathrm{O} / \mathrm{H})_{N}$ and $(\mathrm{O} / \mathrm{H})_{R}$ abundances in the MaNGA galaxies are also close to each other. At the same time, the excitation parameter $P$ and the $\mathrm{N}_{2} / \mathrm{R}_{2}$ line ratio for the spaxels with galactocentric distances from 0.775 to $0.825 R_{g}$ are located around the trend described by the $\mathrm{H}$ II regions in which the $(\mathrm{O} / \mathrm{H})_{N}$ abundances are higher than the $(\mathrm{O} / \mathrm{H})_{R}$ abundances by $0.03-0.09$ dex. Therefore, the $(\mathrm{O} / \mathrm{H})_{N}$ values at the optical radii of the MaNGA galaxies are systematically higher than the $(\mathrm{O} / \mathrm{H})_{R}$ abundances. This provides strong evidence suggesting that the discrepancy between the $(\mathrm{O} / \mathrm{H})_{N}$ and $(\mathrm{O} / \mathrm{H})_{R}$ abundances in the peripheral regions of the MaNGA galaxies is caused by the fact that the 1D N-calibration produces incorrect abundances in the spaxels at the peripheral regions of the MaNGA galaxies because the excitation $P$ and/or the nitrogen-to-oxygen abundance ratio $\mathrm{N} / \mathrm{O}$ in those spaxels differ systematically from those 

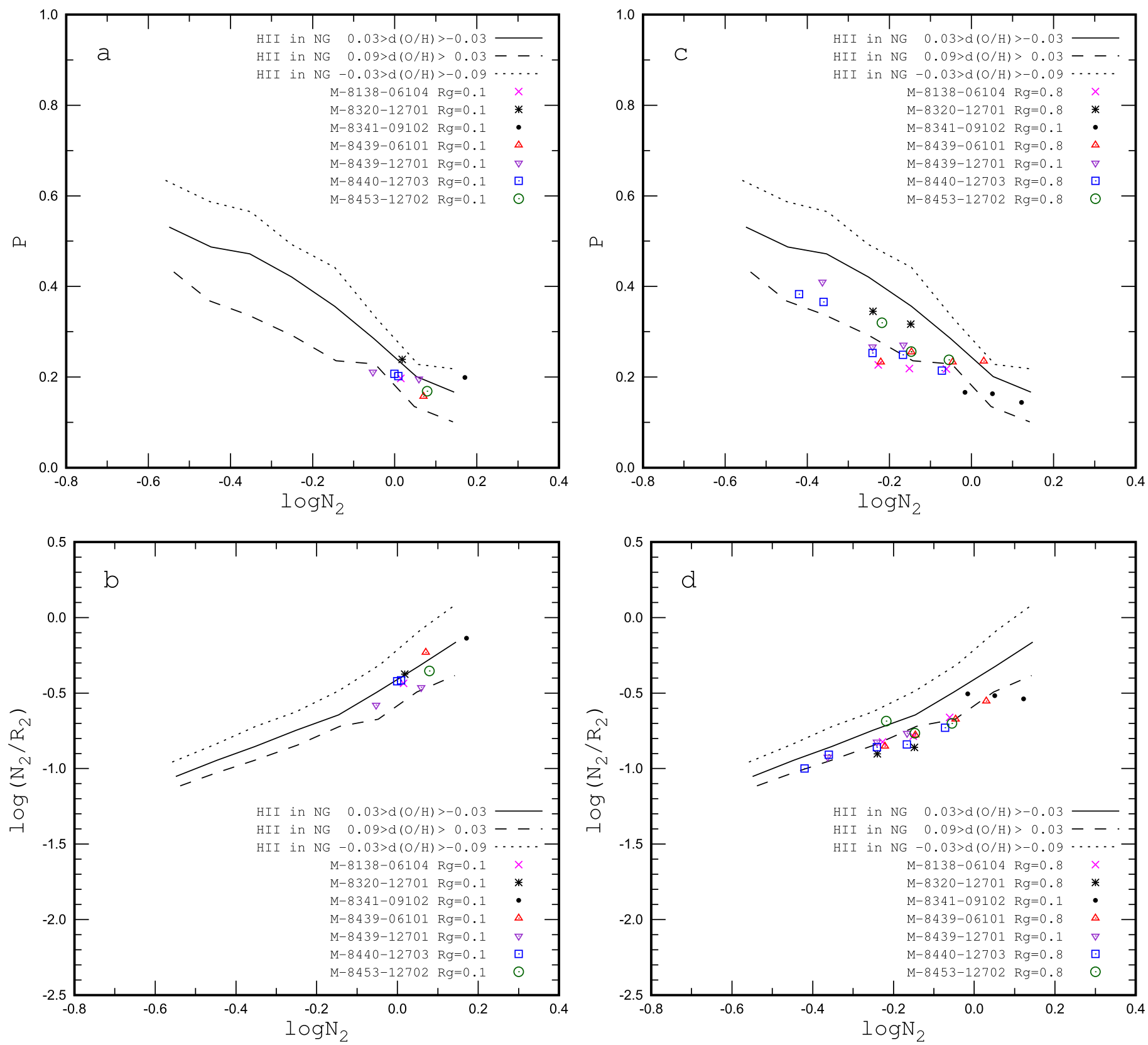

Fig. 11. Panel $a$ : symbols show the excitation parameter $P$ as a function of the $\mathrm{N}_{2}$ line intensity for the spaxels in central regions $\left(R_{g}<0.1\right)$ of several MaNGA galaxies. The mean values of $P$ for objects in bins of $0.1 \mathrm{dex}$ in $\log \mathrm{N}_{2}$ are presented. The solid line shows the excitation parameter $P$ as a function of $\mathrm{N}_{2}$ for the $\mathrm{H}$ II regions in the nearby galaxies with $0.03>\log (\mathrm{O} / \mathrm{H})_{N}-\log (\mathrm{O} / \mathrm{H})_{R}>-0.03$. The long-dashed line shows $P$ for $\mathrm{H}$ II regions with $0.09>\log (\mathrm{O} / \mathrm{H})_{N}-\log (\mathrm{O} / \mathrm{H})_{R}>0.03$, and the short-dashed line shows $P$ for $\mathrm{H}$ II regions with $-0.03>\log (\mathrm{O} / \mathrm{H})_{N}-$ $\log (\mathrm{O} / \mathrm{H})_{R}>-0.09$. Panel $b$ : same as panel $a$ but for the $\mathrm{N}_{2} / \mathrm{R}_{2}$ line ratio. Panel $c$ shows the same as panel $a$ but for spaxels with galactocentric distances from 0.775 to $0.825 R_{g}$. Panel $d$ shows the same as panel $b$ but for spaxels with galactocentric distances from 0.775 to $0.825 R_{g}$.

values in the calibrating points with similar $(\mathrm{O} / \mathrm{H})_{N}$ and $(\mathrm{O} / \mathrm{H})_{R}$ abundances. Ho et al. (2015) also noted that the radial change of oxygen abundances derived with two different $1 \mathrm{D}$ calibrations can differ significantly when the ionization parameters change systematically with radius.

Panel c of Fig. 10 shows the comparison between the $(\mathrm{O} / \mathrm{H})_{P}$ and $(\mathrm{O} / \mathrm{H})_{R}$ abundances for our sample of MaNGA galaxies. The circles mark the central abundances; the plus symbols depict the abundances at the optical radii $R_{25}$. Unfortunately, the $(\mathrm{O} / \mathrm{H})_{P}$ at the optical radius $R_{25}$ can be estimated in a few MaNGA galaxies only (see above). Again, the solid line shows $(\mathrm{O} / \mathrm{H})_{P}$ as a function of $(\mathrm{O} / \mathrm{H})_{R}$ abundance for our sample of $\mathrm{H}$ II regions in nearby galaxies, and the long-dashed line shows the oxygen abundances for those $\mathrm{H}$ II regions obtained with the lines $\mathrm{R}_{2}$ and $\mathrm{N}_{2}$ increased by a factor of 1.3. Panel c of Fig. 10 shows that the central $(\mathrm{O} / \mathrm{H})_{P}$ and $(\mathrm{O} / \mathrm{H})_{R}$ abundances in the MaNGA galaxies follow the trend described by the $\mathrm{H}$ II regions in nearby galaxies. This is additional evidence suggesting that the DIG contribution to the MaNGA spaxel spectra cannot be large, that is, the enhancement of the $\mathrm{R}_{2}$ and $\mathrm{N}_{2}$ strengths does not exceed a factor of $\sim 1.3$.

The $1 \mathrm{D} \mathrm{N} \mathrm{N}_{2}$ calibration suggested by Pettini \& Pagel (2004),

$12+\log (\mathrm{O} / \mathrm{H})_{\mathrm{PP} 04, \mathrm{~N}_{2}}=8.90+0.57 \times N_{2}$, 

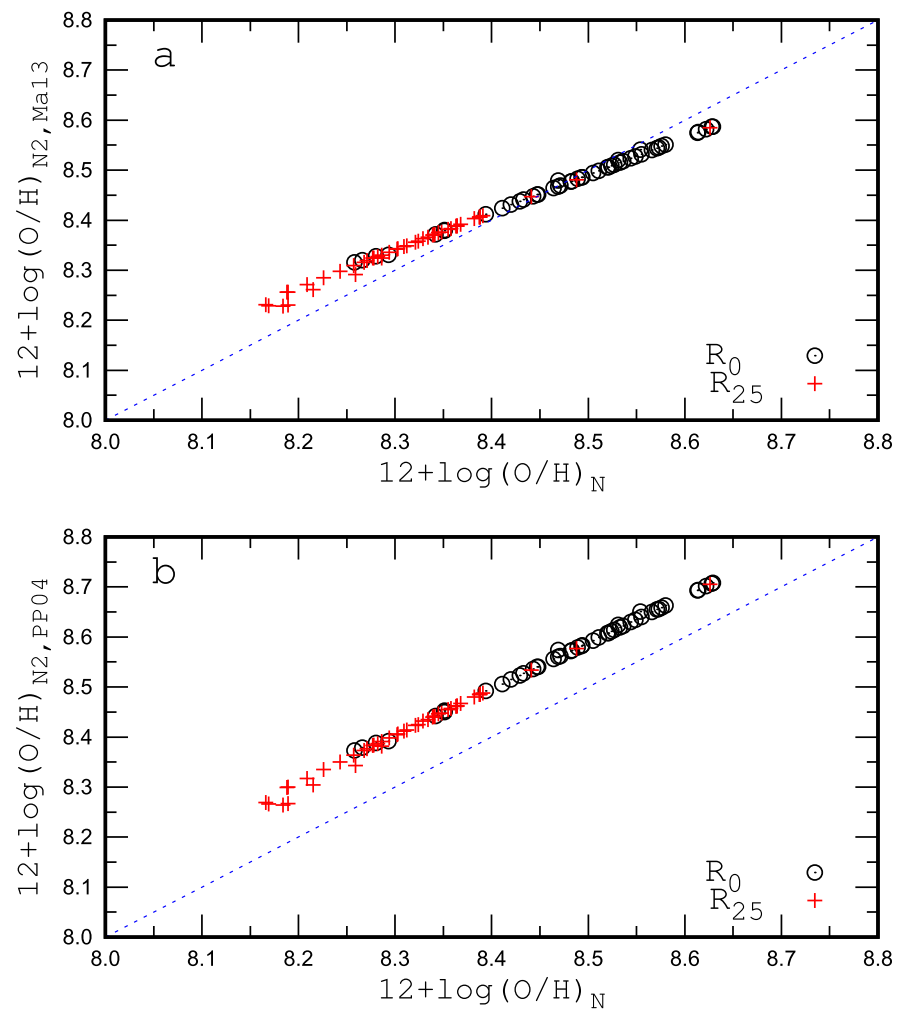

Fig. 12. Comparison of the oxygen abundances estimated through different $\mathrm{N}_{2}$ calibration relations. Panel a: $(\mathrm{O} / \mathrm{H})_{N_{2}, M a 13}$ abundances obtained through the N2 calibration relation of Marino et al. (2013) versus the $(\mathrm{O} / \mathrm{H})_{N}$ abundances determined using our expression for the $N$ calibration. The circles indicate the central (intersect) oxygen abundances for our sample of MaNGA galaxies. The plus symbols show abundances (intersect values) at the optical radius $R_{25}$. The line is that of equal values. Panel $b$ : same as the upper panel but for the $(\mathrm{O} / \mathrm{H})_{N_{2}, P P 04}$ abundances obtained through the $\mathrm{N}_{2}$ calibration relation of Pettini \& Pagel (2004).

and the updated version of the calibration relation suggested by Marino et al. (2013),

$12+\log (\mathrm{O} / \mathrm{H})_{\mathrm{Ma} 13, \mathrm{~N} 2}=8.743+0.462 \times N_{2}$,

where

$N_{2}=\log \left(\frac{[\mathrm{N} \mathrm{II}] \lambda 6584}{\mathrm{H} \alpha}\right)$,

are widely used for abundance estimations. Using these relations, we also estimated the $(\mathrm{O} / \mathrm{H})_{\mathrm{PP} 04, \mathrm{~N} 2}$ and $(\mathrm{O} / \mathrm{H})_{\mathrm{Ma}_{13, \mathrm{~N}_{2}}}$ abundances in the MaNGA galaxies.

Panel a of Fig. 12 shows a comparison of the $(\mathrm{O} / \mathrm{H})_{N 2, M a 13}$ abundances obtained through the $\mathrm{N}_{2}$ calibration relation of Marino et al. (2013) as a function of the $(\mathrm{O} / \mathrm{H})_{N}$ abundances determined through our expression of the $N$ calibration for our sample of MaNGA galaxies; the circles indicate the central (intersect) oxygen abundances. The plus symbols indicate abundances (intersect values) at the optical radii $R_{25}$. The lower panel shows the same as the upper panel but for the $(\mathrm{O} / \mathrm{H})_{N_{2}, P P 04}$ abundances obtained through the $\mathrm{N}_{2}$ calibration relation of Pettini \& Pagel (2004). Inspection of Fig. 12 reveals that the oxygen abundances estimated using the same abundance indicator depend significantly on the calibration relations (on the sample of the calibrating data points).
Thus, the $R$ calibration produces the most reliable oxygen abundances in the MaNGA galaxies.

The $N$ calibration yields reliable oxygen abundances for spaxels where the excitation $P$ and the nitrogen-to-oxygen abundance ratio are similar to those values in the calibrating points located close to the $\mathrm{O} / \mathrm{H}=f\left(\mathrm{~N}_{2}\right)$ relation in the $(\mathrm{O} / \mathrm{H})_{R}-\mathrm{N}_{2}$ diagram (this holds for the central parts of the MaNGA galaxies), but produces incorrect abundances for spaxels where the excitation $P$ and/or the nitrogen-to-oxygen abundance ratio differs from the those values in the calibrating points (in the outer parts of the MaNGA galaxies).

The $S$ calibration results in underestimated oxygen abundances in the MaNGA galaxies since the sulphur line $S_{2}$ is increased in the MaNGA spectra by a factor of up to $\sim 2$ as compared to its counterpart in the $\mathrm{H}$ II regions in nearby galaxies of a given value of $\mathrm{N}_{2}$.

The central oxygen abundance and the radial abundance gradient obtained for each target galaxy using the $R$ calibration are reported in Table 1.

\section{Discussion}

Zhang et al. (2017) report that the strength of the low-ionization lines [N II], [O II], and [S II] relative to the Balmer lines increases with decreasing $\mathrm{H} \alpha$ surface brightness ( $\mathrm{H} \alpha$ fluxes in spaxels) in the MaNGA galaxies at a fixed galactocentric distance. At the same time, the [O III] intensity does not change with the $\mathrm{H} \alpha$ surface brightness. Zhang et al. (2017) concluded that the radiation of the DIG is dominant in the spectra of spaxels with low $\mathrm{H} \alpha$ fluxes. Thus, on the one hand, the DIG contribution to the spaxel spectra may be responsible for the difference between the properties (e.g. excitation) of the spectra of H II regions in nearby galaxies and the spaxel spectra of MaNGA galaxies. On the other hand, the excitations of the SDSS fibre spectra are close to the excitation of the spectra of $\mathrm{H}$ II regions in nearby galaxies while the excitation of the MaNGA spaxel spectra differ from that in the $\mathrm{H}$ II regions and SDSS fibre spectra (panel d of Fig. 6). Here we examine whether or not the DIG contribution is responsible for the difference between the properties of the spectra of $\mathrm{H} \mathrm{II}$ regions in nearby galaxies and the spaxel spectra of MaNGA galaxies.

One might expect from the general consideration that the $[\mathrm{N} \mathrm{II}] / \mathrm{H} \alpha$ in the spaxel spectrum depends on the nitrogen abundance (or on the oxygen abundance and the nitrogen-to-oxygen abundance ratio) and on the excitation parameter $P$. Does the global change of $[\mathrm{N} \mathrm{II}] / \mathrm{H} \alpha$ with galactocentric distance in a galaxy correlate with the change of the $\mathrm{H} \alpha$ surface brightness? Panel a of Fig. 13 shows the $[\mathrm{N} \mathrm{II}] / \mathrm{H} \alpha$ as a function of galactocentric distance in the discs of three MaNGA galaxies. Panel $b$ of Fig. 13 shows the $\mathrm{H} \alpha$ flux $F_{\mathrm{H} \alpha}$ in individual spaxels as a function of galactocentric distance in the discs of those galaxies. The mean values in bins of $0.1 R_{g}$ are presented. Since only a relative comparison of the $\mathrm{H} \alpha$ fluxes across the disc of a galaxy is performed, the measured (and dereddened) $\mathrm{H} \alpha$ fluxes in units of $10^{-17} \mathrm{erg} / \mathrm{s} / \mathrm{cm}^{2} / \AA /$ spaxel can be used instead of the fluxes reduced to the galaxy's distance. Because the aperture is the same for all the spaxels, the $\mathrm{H} \alpha$ flux in spaxels can be used as the $\mathrm{H} \alpha$ surface brightness (in arbitrary units). Figure 13 shows that the changes of the $[\mathrm{N} \mathrm{II}] / \mathrm{H} \alpha$ ratio along the radius are rather similar in the galaxies studied, while the changes of $\mathrm{H} \alpha$ fluxes are significantly different. This suggests that $[\mathrm{N}$ II $] / \mathrm{H} \alpha$ depends more strongly on other parameters than on the $\mathrm{H} \alpha$ flux. 

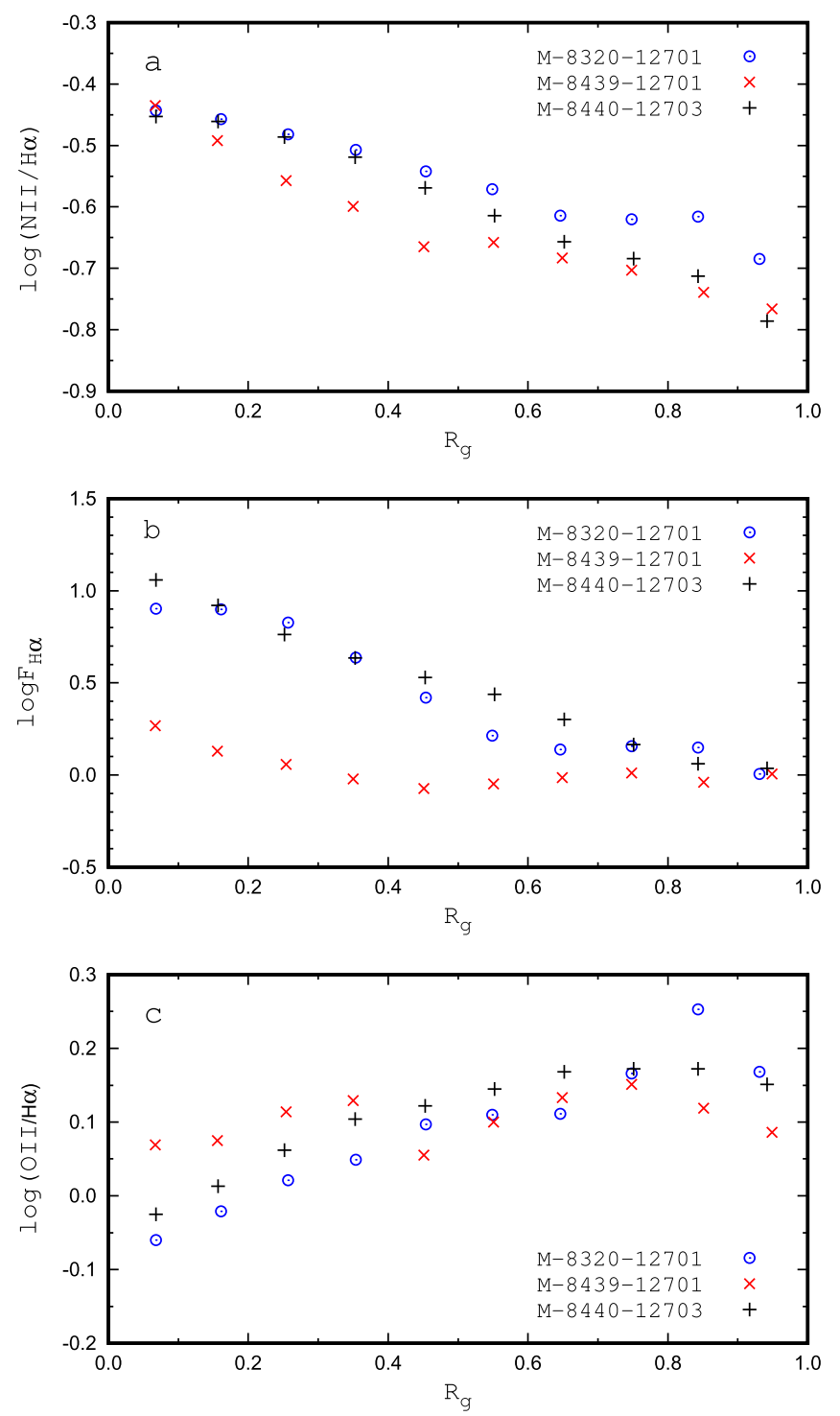

Fig. 13. $[\mathrm{N} I I] / \mathrm{H} \alpha$ line ratio (panel $a$ ), the $\mathrm{H} \alpha$ flux (panel $b$ ), and the $[\mathrm{O} \mathrm{II}] / \mathrm{H} \alpha$ line ratio (panel $c$ ) as a function of galactocentric distance in the discs of three MaNGA galaxies. The mean values in bins of $0.1 R_{g}$ are presented.

Panel c of Fig. 13 shows the $[\mathrm{O} \mathrm{II}] / \mathrm{H} \alpha$ ratio as a function of galactocentric distance in the discs of three MaNGA galaxies. The change of $[\mathrm{O} \mathrm{II}] / \mathrm{H} \alpha$ with galactocentric distance shows the opposite trend in comparison to the change of $[\mathrm{O} \mathrm{II}] / \mathrm{H} \alpha$, that is, the $[\mathrm{O} \mathrm{II}] / \mathrm{H} \alpha$ increases with galactocentric distance while the $[\mathrm{N} \mathrm{II}] / \mathrm{H} \alpha$ decreases. Inspection of Fig. 13 suggests again that the $[\mathrm{O}$ II $] / \mathrm{H} \alpha$ ratio not only depends on the $\mathrm{H} \alpha$ flux but also on other parameters.

Figure 14 shows the $[\mathrm{N}$ II]/H $\alpha$ (panel a), [O II]/H $\alpha$ (panel b) line ratios, and the excitation parameter $P$ (panel c) as a function of $\mathrm{H} \alpha$ flux for spaxels with galactocentric distances $0.85>R_{g}>$ 0.75 and $0.15>R_{g}>0.05$ for a number of the MaNGA galaxies. The symbols denote the mean values in bins of 0.1 dex in $\mathrm{H} \alpha$ flux. The changes of the $[\mathrm{N} I I] / \mathrm{H} \alpha$ and $\left[\mathrm{O}_{\mathrm{II}}\right] / \mathrm{H} \alpha$ ratios in the spaxels of the central parts of the galaxies $\left(0.15>R_{g}>0.05\right)$ is small. The changes of the $[\mathrm{N} \mathrm{II}] / \mathrm{H} \alpha$ and $[\mathrm{O} \mathrm{II}] / \mathrm{H} \alpha$ ratios in the spaxels in the outer parts of the galaxies $\left(0.85>R_{g}>0.75\right)$ is more appreciable. Again, the change of $[\mathrm{O} \mathrm{II}] / \mathrm{H} \alpha$ with $\mathrm{H} \alpha$ flux shows the opposite trend than the change of $[\mathrm{O} \mathrm{II}] / \mathrm{H} \alpha$, that is,
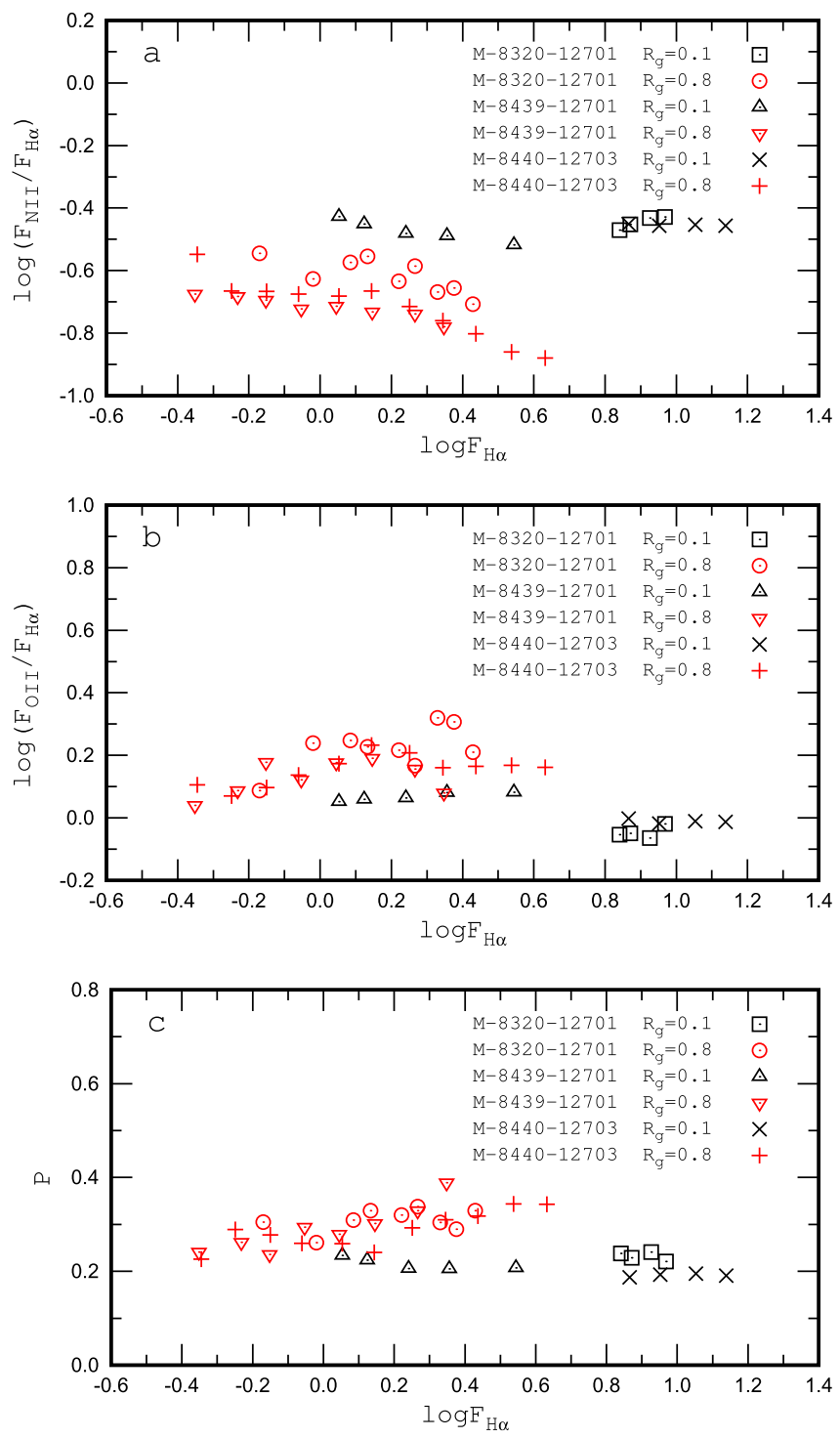

Fig. 14. $[\mathrm{N} I I] / \mathrm{H} \alpha$ (panel $a),[\mathrm{O} \mathrm{II}] / \mathrm{H} \alpha$ (panel $b$ ) line ratios, and the excitation parameter $P$ (panel $c$ ) as a function of $\mathrm{H} \alpha$ flux for spaxels with galactocentric distances $0.85>R_{g}>0.75$ and $0.15>R_{g}>0.05$ for a number of the MaNGA galaxies. The symbols are the mean values in bins of 0.1 dex in the $\mathrm{H} \alpha$ flux.

the $[\mathrm{O}$ II $] / \mathrm{H} \alpha$ ratio increases with $\mathrm{H} \alpha$ flux while the $[\mathrm{N} \mathrm{II}] / \mathrm{H} \alpha$ ratio decreases. Since the excitation $P$ also changes with the $\mathrm{H} \alpha$ flux (panel c of Fig. 14), this may be responsible for the changes of the strengths of the $[\mathrm{N} \mathrm{II}] / \mathrm{H} \alpha$ and $[\mathrm{O} \mathrm{II}] / \mathrm{H} \alpha$ ratios along with the changes of the $\mathrm{H} \alpha$ flux.

The panels in the left column of Fig. 15 show the $[\mathrm{N} \mathrm{II}] / \mathrm{H} \alpha$ ratio as a function of the excitation parameter $P$ for the spaxels in the MaNGA galaxy M-8439-12701 and for the H II regions in nearby galaxies with abundances $12+\log (\mathrm{O} / \mathrm{H}) \pm 0.05$ (the value of $12+\log (\mathrm{O} / \mathrm{H})$ is indicated in each panel). The blue points are spaxels in M-8439-12701 and the red squares are the $\mathrm{H}$ II regions in nearby galaxies. The squares represent the mean values of the $[\mathrm{N} I \mathrm{II}] / \mathrm{H} \alpha$ ratio in bins of 0.05 dex in $P$. The bars denote the mean values of the scatter of $[\mathrm{N} \mathrm{II}] / \mathrm{H} \alpha$ in the bins. The solid line represents the same $\mathrm{H}$ II regions in nearby galaxies but both the $\mathrm{R}_{2}$ and $\mathrm{N}_{2}$ lines are increased by a factor of 1.3. Inspection of Fig. 15 reveals that the $[\mathrm{N} \mathrm{II}] / \mathrm{H} \alpha-$ $P$ diagrams for the MaNGA spaxel spectra are compatible 

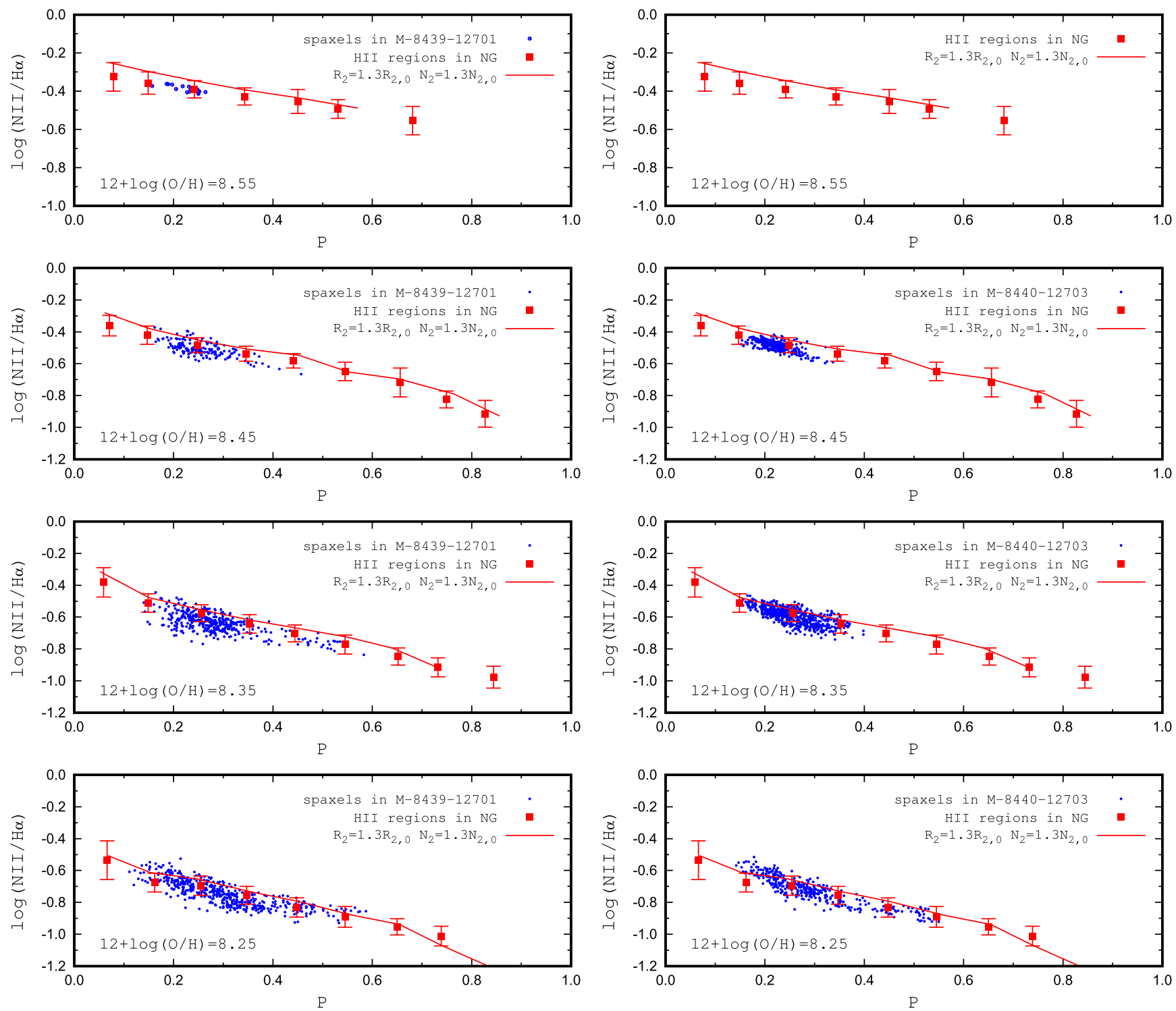

Fig. 15. Left column panels: $[\mathrm{N} \mathrm{II}] / \mathrm{H} \alpha$ as a function of excitation parameter $P$ for the spaxels in the MaNGA galaxy M-8439-12701 and for the $\mathrm{H}$ II regions in nearby galaxies. The blue points are spaxels with abundances $12+\log (\mathrm{O} / \mathrm{H}) \pm 0.05$ in $\mathrm{M}-8439-12701$ (the value of $12+\log (\mathrm{O} / \mathrm{H})$ is indicated in each panel). The red squares are the $\mathrm{H}$ II regions in nearby galaxies with abundances in the same interval. The squares represent the mean values of the $[\mathrm{N} \mathrm{II}] / \mathrm{H} \alpha$ in bins of 0.05 dex in $P$. The bars denote the mean values of the scatter of the $[\mathrm{N}$ II] $/ \mathrm{H} \alpha$ in the bins. The solid line is for the same $\mathrm{H}$ II regions but both the $\mathrm{R}_{2}$ and $\mathrm{N}_{2}$ lines are increased by a factor of 1.3. Right column panels: same for the MaNGA galaxy M-8440-12703.

with the one for the spectra of $\mathrm{H}$ II regions in nearby galaxies. Differences are seen only in low-metallicity, low-excitation regions.

Thus, there is little room for a significant increase of the strength of the low-ionization lines, [O II] and [N II], relative to the Balmer lines in the MaNGA spaxel spectra due to the contribution of the DIG radiation. This suggests that the demarcation line between AGNs and $\mathrm{H}$ II regions in the BPT diagram defined by Kauffmann et al. (2003) is a good criterium to reject the spectra with a significantly distorted strength of the $[\mathrm{N} \mathrm{II}]$ and [O II] lines.

\section{Summary}

The publicly available data obtained by the MaNGA survey form the basis of our current study. We measured the emission lines in the spectrum of each spaxel from the MaNGA datacubes for 47 late-type galaxies. The surface brightness in the SDSS $g$ and $r$ bands was obtained from broadband SDSS images created from the data cube.

It has been suggested that the strength of the low-ionization lines, $\mathrm{R}_{2}, \mathrm{~N}_{2}$, and $\mathrm{S}_{2}$, can be increased (relative to the Balmer lines) in (some) spaxel spectra of the MaNGA survey due to a contribution of the radiation of the DIG and that, consequently, the abundances derived from the spaxel spectra through strongline methods may have large errors. We examine this expectation by comparing the behaviour of the line intensities and the abundances estimated through different calibrations for samples of slit spectra of $\mathrm{H}$ II regions in nearby galaxies, of the fibre spectra from the SDSS, and of the spaxel spectra of the MaNGA survey. We find that the mean value of the $\mathrm{S}_{2}$ strength in the SDSS fibre and MaNGA spaxel spectra is increased significantly (up to a factor of $\sim 2$ ) depending on the metallicity. The mean distortion of $R_{2}$ and $N_{2}$ is less than a factor of $\sim 1.3$. This suggests that 
the demarcation line between AGNs and H II regions in the BPT diagnostic diagram defined by Kauffmann et al. (2003) is a relatively reliable criterium to reject the spectra with significantly distorted strengths of the [N II] and [O II] lines.

We determined and compared the oxygen abundances in the SDSS galaxies through different calibrations based on two catalogues of emission line measurements aiming to estimate the uncertainty in the oxygen abundances due to the uncertainties in the data reduction and line flux measurements. We find that the uncertainty in the abundances are small $(\lessgtr 0.02 \mathrm{dex})$ for objects with metallicity from $12+\log (\mathrm{O} / \mathrm{H}) \sim 8.1$ to $12+\log (\mathrm{O} / \mathrm{H})$ $\sim 8.55$ and are higher for objects outside this interval (with lower and higher metallicities).

We analysed the abundances estimated through different calibrations for samples of the slit spectra of the H II regions in nearby galaxies, of the fibre spectra of the SDSS, and of the spaxel spectra of the MaNGA survey, aiming to establish which calibration produces the most reliable abundances from the MaNGA spaxel spectra.

We find that the 3D $R$ calibration using the $\mathrm{R}_{2}$ and $\mathrm{N}_{2}$ lines produces the most reliable oxygen abundances in the MaNGA galaxies; and that the 1D $N$ calibration produces reliable oxygen abundances for spaxels where the excitation $P$ and the nitrogen-to-oxygen abundance ratio are close to those values in the calibrating points located close to the $\mathrm{O} / \mathrm{H}=f\left(\mathrm{~N}_{2}\right)$ relation in the $(\mathrm{O} / \mathrm{H})_{R}-\mathrm{N}_{2}$ diagram (i.e. in calibrating $\mathrm{H}$ II regions with similar $(\mathrm{O} / \mathrm{H})_{N}$ and $(\mathrm{O} / \mathrm{H})_{R}$ abundances $)$, but produces incorrect abundances for spaxels where the excitation $P$ and/or the nitrogen-to-oxygen abundance ratio differ from the values in the calibrating points.

We determined the abundance distributions (and surface brightness profiles) within the optical radii of the discs of 47 galaxies based on the spaxel spectra from the MaNGA survey. Those data will be used in a forthcoming paper.

Acknowledgements. We are grateful to the referee for his/her constructive comments. L.S.P., E.K.G., and I.A.Z. acknowledge support within the framework of Sonderforschungsbereich (SFB 881) on "The Milky Way System" (especially subproject A5), which is funded by the German Research Foundation (DFG) L.S.P. and I.A.Z. are grateful for the hospitality of the Astronomisches RechenInstitut at Heidelberg University, where part of this investigation was carried out I.A.Z. acknowledges the support of the Volkswagen Foundation under the Trilateral Partnerships grant No. 90411. This work was partly funded by the subsidy allocated to Kazan Federal University for the state assignment in the sphere of scientific activities (L.S.P.). We acknowledge the use of the HyperLeda database (http: //leda.univ-lyon1.fr). This research made use of Montage, funded by the National Aeronautics and Space Administration's Earth Science Technology Office, Computational Technnologies Project, under Cooperative Agreement Number NCC5-626 between NASA and the California Institute of Technology. The code is maintained by the NASA/IPAC Infrared Science Archive. Funding for SDSS-III has been provided by the Alfred P. Sloan Foundation, the Participating Institutions, the National Science Foundation, and the U.S. Department of Energy Office of Science. The SDSS-III web site is http://www . sdss3.org/ SDSS-III is managed by the Astrophysical Research Consortium for the Participating Institutions of the SDSS-III Collaboration including the University of Arizona, the Brazilian Participation Group, Brookhaven National Laboratory, Carnegie Mellon University, University of Florida, the French Participation Group, the German Participation Group, Harvard University, the Instituto de Astrofisica de Canarias, the Michigan State/Notre Dame/JINA Participation Group, Johns Hopkins University, Lawrence Berkeley National Laboratory, Max Planck Institute for Astrophysics, Max Planck Institute for Extraterrestrial Physics, New Mexico State University, New York University, Ohio State University, Pennsylvania State University, University of Portsmouth, Princeton University, the Spanish Participation Group, University of Tokyo, University of Utah, Vanderbilt University, University of Virginia, University of Washington, and Yale University. Funding for the Sloan Digital Sky Survey IV has been provided by the Alfred P. Sloan Foundation, the U.S. Department of Energy Office of Science, and the Participating Institutions. SDSS-IV acknowledges support and resources from the Center for High-Performance Computing at the University of Utah. The SDSS web site is www . sdss. org. SDSS-IV is managed by the
Astrophysical Research Consortium for the Participating Institutions of the SDSS Collaboration including the Brazilian Participation Group, the Carnegie Institution for Science, Carnegie Mellon University, the Chilean Participation Group, the French Participation Group, Harvard-Smithsonian Center for Astrophysics, Instituto de Astrofísica de Canarias, The Johns Hopkins University, Kavli Institute for the Physics and Mathematics of the Universe (IPMU) / University of Tokyo, Lawrence Berkeley National Laboratory, Leibniz Institut für Astrophysik Potsdam (AIP), Max-Planck-Institut für Astronomie (MPIA Heidelberg), Max-Planck-Institut für Astrophysik (MPA Garching), Max-Planck-Institut für Extraterrestrische Physik (MPE), National Astronomical Observatories of China, New Mexico State University, New York University, University of Notre Dame, Observatário Nacional / MCTI, The Ohio State University, Pennsylvania State University, Shanghai Astronomical Observatory, United Kingdom Participation Group, Universidad Nacional Autónoma de México, University of Arizona, University of Colorado Boulder, University of Oxford, University of Portsmouth, University of Utah, University of Virginia, University of Washington, University of Wisconsin, Vanderbilt University, and Yale University.

\section{References}

Andrews, B. H., \& Martini, P. 2013, ApJ, 765, 140

Baldwin, J. A., Phillips, M. M., Terlevich, R. 1981, PASP, 93, 5

Belfiore, F., Maiolino, R., Bundy, K, et al. 2015, MNRAS, 449, 867

Belfiore, F., Maiolino, R., Tremonti, C., et al. 2017, MNRAS, 469, 151

Blanton, M. R., \& Roweis, S. 2007, AJ, 133, 734

Bresolin, F., Ryan-Weber, E., Kennicutt, R. C., Goddard, Q. 2009, ApJ, 695, 580

Bresolin, F., Kennicutt, R. C., Ryan-Weber, E. 2012, ApJ, 750, 122

Brinchmann, J., Charlot, S., White, S. D. M., et al. 2004, MNRAS, 351, 115

Bruzual, G., \& Charlot, S. 2003, MNRAS, 344, 1000

Bundy, K., Bershady, M. A., Law, D. R., et al. 2015, ApJ, 798, 7

Cardelli, J. A., Clayton, G. C., Mathis, J. S. 1989, ApJ, 345, 245

Cid Fernandes, R., Stasińska, G., Schlickmann, M. S., et al. 2010, MNRAS, 403, 1036

Cowie, L. L., \& Barger, A. J. 2008, ApJ, 686, 72

de Vaucouleurs, G., de Vaucouleurs, A., Corvin, H. G., et al. 1991, Third Reference Catalog of Bright Galaxies, (New York: Springer Verlag)

Edmunds, M. G., \& Pagel, B. E. J. 1978, MNRAS, 185, 77

Erb, D. K., Shapley, A. E., Pettini, M., et al. 2006, ApJ, 644, 813

García-Benito, R., Zibetti, S., Sánchez, S. F., et al. 2015, A\&A, 576, A135

Garnett, D. R. 2002, ApJ, 581, 1019

Goddard, Q. E., Bresolin, F., Kennicutt, R. C., Ryan-Weber, E. V., RosalesOrtega, F. F. 2011, MNRAS, 412, 1246

Grebel, E. K., Gallagher, J. S. III, Harbeck, D. 2003, AJ, 125, 1926

Guseva, N. G., Papaderos, P., Meyer, H. T., Izotov, Y. I., Fricke, K. J. 2009, A\&A, 505,63

Ho, I.-T., Kudritzki, R.-P., Kewley, L. J., et al. 2015, MNRAS, 448, 2030

Husemann, B., Jahnke, K., Sánchez, S. F., et al. 2013, A\&A, 549, A87

Izotov, Y. I., Thuan, T. X., Lipovetsky, V. A. 1994, ApJ, 435, 647

Izotov, Y. I., Guseva, N. G., Fricke, K. J., Henkel, C. 2015, MNRAS, 451, 2251

Karachentsev, I. D., Makarov, D. I., Kaisina, E. I. 2013, AJ, 145, 101

Kauffmann, G., Heckman, T. M., Tremonti, C., et al. 2003, MNRAS, 346, 1055

Kewley, L. J., Dopita, M. A., Sutherland, R. S., Heisler, C. A., Trevena, J. 2001 ApJ, 556, 121

Kudritzki, R.-P., Urbaneja, M. A., Bresolin, F., Hosek, M. W., Przybilla, N. 2014, ApJ, 788, 56

Lee, H., Skillman, E. D., Venn, K. A. 2005, ApJ, 620, 223

Lequeux, J., Peimbert, M., Rayo, J. F., Serrano, A., Torres-Peimbert, S. 1979, A\&A, 80, 155

Maier, C., Lilly, S. J., Ziegler, B. L., et al. 2014, ApJ, 792, 3

Maiolino, R., Nagao, T., Grazian, A., et al. 2008, A\&A, 488, 463

Maraston, C., \& Strömbäck, G. 2011, MNRAS, 418, 2785

Marino, R. A., Rosales-Ortega, F. F., Sánchez, S. F., et al. 2013, A\&A, 559, A114

Muñoz-Mateos, J. C., Sheth, K., Regan, M., et al. 2015, ApJS, 219, 3

Patterson, M. T., Walterbos, R. A. M., Kennicutt, R. C., Chiappini, C., Thilker,

D. A. 2012, MNRAS, 422, 401

Petrosian, V. 1976, ApJ, 209, L1

Pettini, M., \& Pagel, B. E. J. 2004, MNRAS, 348, L59

Pilyugin, L. S. 2000, A\&A, 362, 325

Pilyugin, L. S. 2001, A\&A, 369, 594

Pilyugin, L. S., \& Grebel, E. K. 2016, MNRAS, 457, 3678

Pilyugin, L. S., \& Thuan, T. X. 2005, ApJ, 631, 231

Pilyugin, L. S., \& Thuan, T. X. 2011, ApJ, 726, 23

Pilyugin, L. S., Grebel, E. K., Mattsson, L. 2012, MNRAS, 424, 2316

Pilyugin, L. S., Lara-López, M. A., Grebel, E. K., et al. 2013, MNRAS, 432, 1217

Pilyugin, L. S., Grebel, E. K., Kniazev, A. Y. 2014, AJ, 147, 131 


\section{S. Pilyugin et al.: Abundances based on spaxel spectra of the MaNGA survey}

Pilyugin, L. S., Grebel, E. K., Zinchenko, I. A., Nefedyev, Y. A., Vílchez, J. M. 2017, A\&A, 608, A127

Sánchez, S. F., Kennicutt, R. C., Gil de Paz, A., et al. 2012a, A\&A, 538, A8

Sánchez, S. F., Rosales-Ortega, F. F., Marino, R. A., et al. 2012b, A\&A, 546, A2

Sánchez, S. F., Rosales-Ortega, F. F., Iglesias-Paramo, J., et al. 2014, A\&A, 563, A49

Sánchez-Menguiano, L., Sánchez, S. F., Pérez, I., et al. 2016, A\&A, 587, A70

Sanders, R. L., Shapley, A. E., Zhang, K., Yan, R. 2017, ApJ, 850, 136

Schlafly, E. F., \& Finkbeiner, D. P. 2011, ApJ, 737, 103

SDSS Collaboration, 2017, ApJS, 233, 25
Steidel, C. C., Rudie, G. C., Strom, A. L., et al. 2014, ApJ, 795, 165 Storey, P. J., \& Zeippen, C. J. 2000, MNRAS, 312, 813

Thomas, D., Maraston, C., Johansson, J. 2011, MNRAS, 412, 2183

Thuan, T. X., Pilyugin, L. S., Zinchenko, I. A. 2010, ApJ, 712, 1029

Tremonti, C. A., Heckman, T. M., Kauffmann, G., et al. 2004, ApJ, 613, 898

Whitford, A. E. 1958, AJ, 63, 201

Zahid, H. J., Geller, M. J., Kewley, L. J., et al. 2013, ApJ, 771, L19

Zaritsky, D., Kennicutt, R. C., Huchra, J. P. 1994, ApJ, 420, 87

Zhang, K., Yan, R., Bundy, K., et al. 2017, MNRAS, 466, 3217

Zinchenko, I. A., Pilyugin, L. S., Grebel, E. K., Sánchez, S. F., Vílchez, J. M. 2016, MNRAS, 462, 2715 